\title{
NUMERICAL SIMULATION OF TURBULENT CONVECTIVE FLOW OVER WAVY TERRAIN
}

\author{
A. DÖRNBRACK and U. SCHUMANN \\ DLR, Institute of Atmospheric Physics, D-82230 Oberpfaffenhofen, Germany
}

(Received in final form 23 December, 1992)

\begin{abstract}
By means of a large-eddy simulation, the convective boundary layer is investigated for flows over wavy terrain. The lower surface varies sinusoidally in the downstream direction while remaining constant in the other. Several cases are considered with amplitude $\delta$ up to $0.15 H$ and wavelength $\lambda$ of $H$ to $8 H$, where $H$ is the mean fluid-layer height. At the lower surface, the vertical heat flux is prescribed to be constant and the momentum flux is determined locally from the Monin-Obukhov relationship with a roughness length $z_{0}=10^{-4} \mathrm{H}$. The mean wind is varied between zero and $5 w_{*}$, where $w_{*}$ is the convective velocity scale. After rather long times, the fiow structure shows horizontal scales up to $4 H$, with a pattern similar to that over flat surfaces at corresponding shear friction. Weak mean wind destroys regular spatial structures induced by the surface undulation at zero mean wind. The surface heating suppresses mean-flow recirculation-regions even for steep surface waves. Short surface waves cause strong drag due to hydrostatic and dynamic pressure forces in addition to frictional drag. The pressure drag increases slowly with the mean velocity, and strongly with $\delta / H$. The turbulence variances increase mainly in the lower half of the mixed layer for $U / w_{*}>2$.
\end{abstract}

\section{Introduction}

In this paper, we investigate the effect of wavy terrain and mean wind on the fully turbulent convective boundary layer (CBL) by means of large-eddy simulation (LES). The objective is to identify the flow changes induced by hilly terrain with amplitude smaller than, and wavelengths comparable to, the depth of the CBL. The results should be of interest for parameterization of drag over small-scale topography (Hunt et al., 1991). As a prototype of the wide variety of hilly terrain shapes, we consider surfaces with height varying sinusoidally in one direction only. A constant mean wind is imposed, blowing perpendicular to the wave crests. The surface is uniformly heated. The top of the CBL is bounded by an adiabatic and free-slip lid which should approximate a very strong capping inversion.

The CBL has been the subject of extensive numerical simulations. Most studies concentrated on homogeneous and flat surfaces; for example, Schmidt and Schumann (1989) investigated the CBL at zero mean wind, where the flow structure exhibits strong isolated updrafts surrounded by areas of weaker downward motion. The results were used, inter alia, to determine the heat transfer at the surface (Schumann, 1988). Sykes and Henn (1989) showed that shear causes longitudinal rolls within the $\mathrm{CBL}$ if the friction velocity at the surface exceeds about 0.35 of the convective velocity scale. Recently, Krettenauer and Schumann (1992) and Walko et al. (1992) investigated the CBL over wavy terrain at zero mean wind. Coherent rolls parallel to the wave crests show maximum motion amplitudes if 
the surface wavelength equals about four times the depth of the CBL (Schumann, 1991b). Variations in surface heating over flat terrain enforce motion structures similar to those over wavy terrain for zero wind speed (Hadfield et al., 1991; Schumann, 1991b) but light wind weakens the circulation (Hechtel et al,, 1989; Hadfield et al., 1992). The impact of moderately strong winds in combination with wavy terrain on the three-dimensional structure of turbulent motions has not yet been investigated numerically for the CBL.

However, several analytical (e.g., Hunt et al., 1988) and numerical (Taylor, 1977; Mason and King, 1984; Zeman and Jensen, 1987) studies considered flow over wavy terrain with neutral stratification and the results of such studies have been used to develop parameterizations for the drag induced by small-scale topography (Taylor et al., 1989; Emeis, 1990). The analytical theories are restricted to 'gentle' hills without separation and the numerical studies treat two-dimensional flows with statistical turbulence models. Results from LES show, however, that the CBL tends to form roll motions with axes both parallel and perpendicular to the surface waves, together with more irregular turbulent motion components, so that three-dimensional simulations are necessary. Moreover, the horizontal scales of turbulent motions are typically up to four times larger than the depth of the boundary layer so that statistical models are questionable for shorter surface wavelengths. Mason (1987) finds that an increase of the mixing length causes a strong reduction of the range of regions with separated flows. As reported in Hunt et al. (1991), inclusion of horizontal diffusion into two-dimensional models caused the 'form' drag (dynamic pressure forces at the surface) to increase by approximately $60 \%$, even for gentle slopes. These facts signify the importance of large horizontal scales.

Laboratory studies of flow over wavy terrain have been limited to zero heat flux at the surface. Buckles et al. (1984) investigated the flow for neutral stability at moderate Reynolds numbers and found a large separated region with recirculating flow in the valley of the surface waves for an amplitude of $10 \%$ of the wavelength. Taylor et al. (1987) reviewed field experiments on boundary layers over low hills for near-neutral static stability; see also Hunt et al. (1991). Experimental results on flow over hilly terrain are given by Mason and King (1984) and Grant and Mason (1990). Mason and King (1984) suggest that rather steep slopes are required for separation over smooth surfaces but find clear separation for valley slopes of $32^{\circ}$ to the horizontal for a roughness to wave-amplitude ratio of about $10^{-4}$. Increased mixing due to convective flow and larger surface roughness inhibits separation (Tampieri, 1987) but upslope flows induced by one-sided valley heating may enhance separation (Scorer, 1978; Mason, 1987). Grant and Mason (1990) show that the roughness length may be generalized to parameterize the surface stress in complex terrain and that the structure of the turbulent boundary layer, for near neutral stability, is remarkably similar to that over flat homogeneous surfaces. However, turbulence data are shown only for neutral and stable atmospheres because of statistical sampling problems associated with convective flows. 
In the day-time atmosphere, convective conditions are often important. Only a few numerical two-dimensional studies have been performed for such conditions (Mason, 1987). Field observations of the CBL over irregular but gentle hilly terrain have been reported by Kaimal et al. (1982), Druilhet et al. (1983) and Huynh et al. (1990). Generally, the changes in the turbulence variances are found to be small compared to the statistical uncertainties of such measurements where one compares data of flat and 'gently rolling terrain'. For a 'rugged' terrain with amplitudes of $50 \mathrm{~m}$ and instances between the ridges of order $1 \mathrm{~km}$, Brutsaert and Kustas (1987) found that the relative importance of mechanical turbulence as compared to convective turbulence was much larger than over smoother terrain for the same degree of atmospheric instability. Brutsaert and Sugita (1990) used data from hilly terrain with typical wavelength of 0.5 to $1 \mathrm{~km}$ and wave amplitude of $25 \mathrm{~m}$, and showed that the Monin-Obukhov similarity is applicable for temperature up to about 0.1 times the depth of the CBL. For very strong winds, the form drag increases approximately quadratically with slope steepness (Taylor et al., 1989) while frictional drag and heat and mass transfer remain unchanged, at least for gentle terrain (Raupach et al., 1992). It is not yet clear how these results transfer into the mixed sheared/convective situation.

This study is limited to a very idealized form of hilly terrain. This should help to isolate the basic effects. The lid at the top of the CBL is taken to guarantee the existence of a truly steady state and to avoid dependence on the details of the inversion parameters. Moreover, the flow is driven by an uniform horizontal pressure gradient which is determined such that the average of the horizontal velocity across the $\mathrm{CBL}$ is constant. Coriolis forces are neglected. The simplifications could be abandoned in future studies with the numerical method used, but are introduced here to have clearly defined conditions.

We apply LES to simulate the turbulent convection. This approach has been shown to give reliable results for cases with horizontal and inclined flat surfaces by several previous studies; see Schmidt and Schumann (1989), Schumann (1990) and the comparison of four different LES methods in Nieuwstadt et al. (1992). For wavy terrain, the method has been tested for nonlinear but laminar convection in Krettenauer and Schumann (1989). The results show that the method is applicable at least for convective conditions where exchange of momentum by friction at the surface is of relatively small importance compared to internal momentum transport and pressure forces. LES for neutral static stability would require very fine grid resolution near the surface because of very thin surface shear layers (Mason and King, 1984; Hunt et al., 1988) with strong variations in turbulence profiles (Zeman and Jensen, 1987). This study does not resolve that inner layer but concentrates on the effect of the surface on the turbulence in the mixed layer. It relies on the assumption that the details of the surface layer of the CBL (for constant surface heat flux) have small impact on the turbulent flow within the CBL. Some qualitative arguments will be given to support this assumption. 


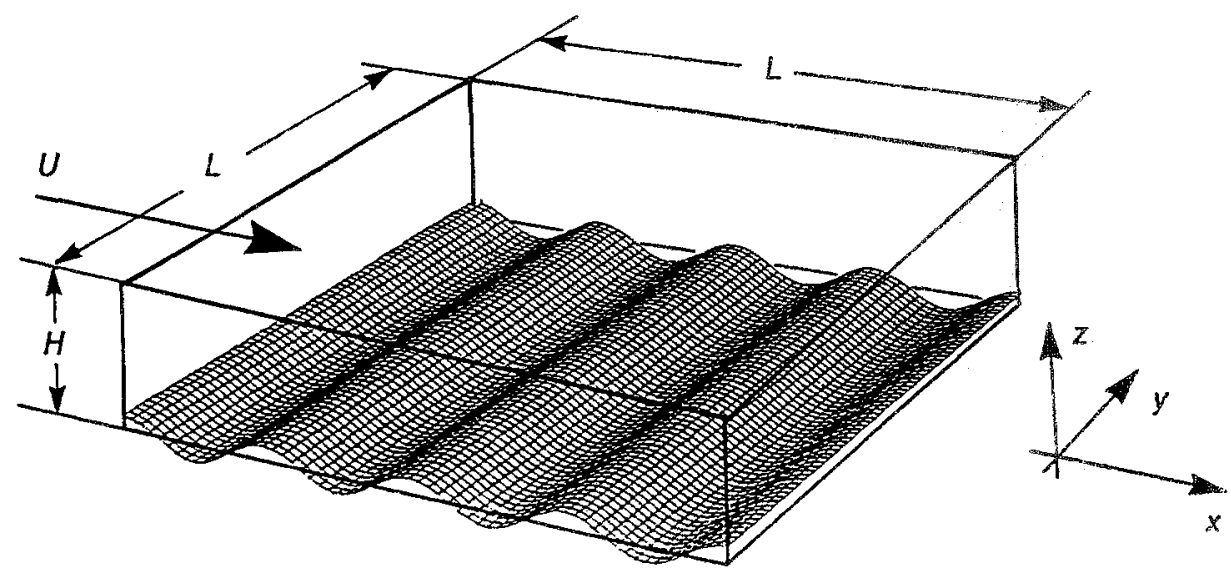

Fig. 1. Perspective view of the computational domain in three dimensions showing the sinusoidai surface-wave in the $x$-direction; the surface height is constant in the $y$-direction. In the example, the wavelength is $\lambda=H$, the wave-amplitude is $\delta=0.1 H$, and the lateral domain-size is $L=4 H$. The mean

flow is in the $x$-direction. The reference case of this study uses $L=8 H$.

\section{The Method}

The LES method is as described in detail in Krettenauer and Schumann (1992). Only its essential properties are repeated here. The numerical scheme integrates the full primitive equations of motion in their non-hydrostatic form together with the temperature equation employing the Boussinesq approximation in three dimensions and as a function of time. The subgrid-scale (SGS) fluxes are determined by means of eddy diffusivities as a function of the grid scale and the square root of the SGS kinetic energy. The latter is computed from a separate transport equation including shear and buoyancy forcing and dissipation. The equations of motion are formulated for the Cartesian velocity components $(u, v, w)$ as a function of curvilinear coordinates $(\bar{x}, \bar{y}, \bar{z})$ which are related to the Cartesian coordinates $(x, y, z)$ according to the transformation $\bar{x}=x, \bar{y}=y, \bar{z}=\eta(x, y, z)$. Here,

$$
\eta=H \frac{(z-h)}{(H-h)}
$$

maps the domain above the wavy surface at height $h(x, y)=\delta \cos (2 \pi x / \lambda)$ and below a plane top surface at $z=H$ onto a rectangular transformed domain. The amplitude $\delta$ and the wavelength $\lambda$ relative to the mean depth $H$ and the lateral domain size $L$ form the essential free geometric parameters of this study; see Figure 1.

The differential equations are approximated by finite differences. In space we use an equidistant staggered grid with equal grid spacings $\Delta x=\Delta y$ in the horizontal directions and $\Delta z$ in the vertical direction of the transformed coordinates. Except for parameter studies, we use $\Delta x=\Delta z$ to ensure resolution of small-scale thermals 
in the surface layer transporting the heat from the bottom surface upwards and of locally isotropic turbulence inside the mixed layer. The momentum equation is integrated using the Adams-Bashforth scheme in time and second-order finite differences in space, and the transport equations for temperature and SGS kinetic energy are integrated using a second-order upwind scheme. The pressure is computed iteratively such that the continuity equation is satisfied after each time step.

At the lateral boundaries, cyclic boundary conditions are used. The sidelengths $L$ in the $x$ - and $y$-directions are taken as equal in order to avoid any predominance of horizontally anisotropic structures. The lateral domain size $L$ has to be large compared to the internal scales of convection. Based on the experience of Krettenauer and Schumann $(1989,1992)$, we expect $L=8 H$ to be large enough in this sense. At the bottom surface, the frictional momentum flux is computed locally from the horizontal velocity in the lowest grid cell using the Monin-Obukhov relationships for a rough surface with roughness height $z_{0}$. The heat flux normal to the surface is prescribed such that the vertical heat flux per horizontal unit area is constant. The integration requires no specific temperature boundary conditions for given surface heat flux. For evaluation of the surface temperature $\Delta T$ relative to the bulk mean temperature, we apply again the Monin-Obukhov relationships assuming the surface roughness for heat transfer to be the same as for momentum. The usage of Monin-Obukhov profile functions relies on local equilibrium conditions which might be questionable for steep surfaces and for coarse vertical grids. The latter will be discussed on the basis of parameter studies with different grid spacings and order-of-magnitude estimates as given in the Appendix. At the rigid top surface, the vertical momentum and heat fluxes are set to zero.

The mean velocity $U$ is defined as the average velocity in the $x$-direction across a $y-z$ plane above the surface wave crests. The bulk mean velocity $U_{b}$, which is the average over the fluid domain, differs from $U$ by $U_{b}=U(H-\delta) / H$. After each time step, the actual mean velocity is tested and a mean pressure gradient in the $x$-direction determined such that $U$ stays constant.

The results are normalized by the convective velocity, height, temperature and time scales,

$$
w_{*}=\left(\beta g Q_{S} H\right)^{1 / 3}, \quad H, \quad T_{*}=Q_{S} / w_{*}, \quad t_{*}=H / w_{*},
$$

respectively. Here, $\beta$ is the volumetric expansion coefficient, $g$ is gravity, $Q_{s}$ is the prescribed vertical temperature flux at the surface, and $H$ is the mean height of the flow domain.

The initial conditions prescribe uniform velocities, constant mean temperature and constant SGS kinetic energy. Small random perturbations are added to the temperature and velocity fields to initiate three-dimensional turbulent motions. Ideally, the computations should run until steady state statistics are obtained. The time required to reach that state in the present applications will be longer, the larger the wavelength $\lambda$, because the time required to smooth horizontal variations increases with $\lambda$. For that reason we shall check some statistics and run the 


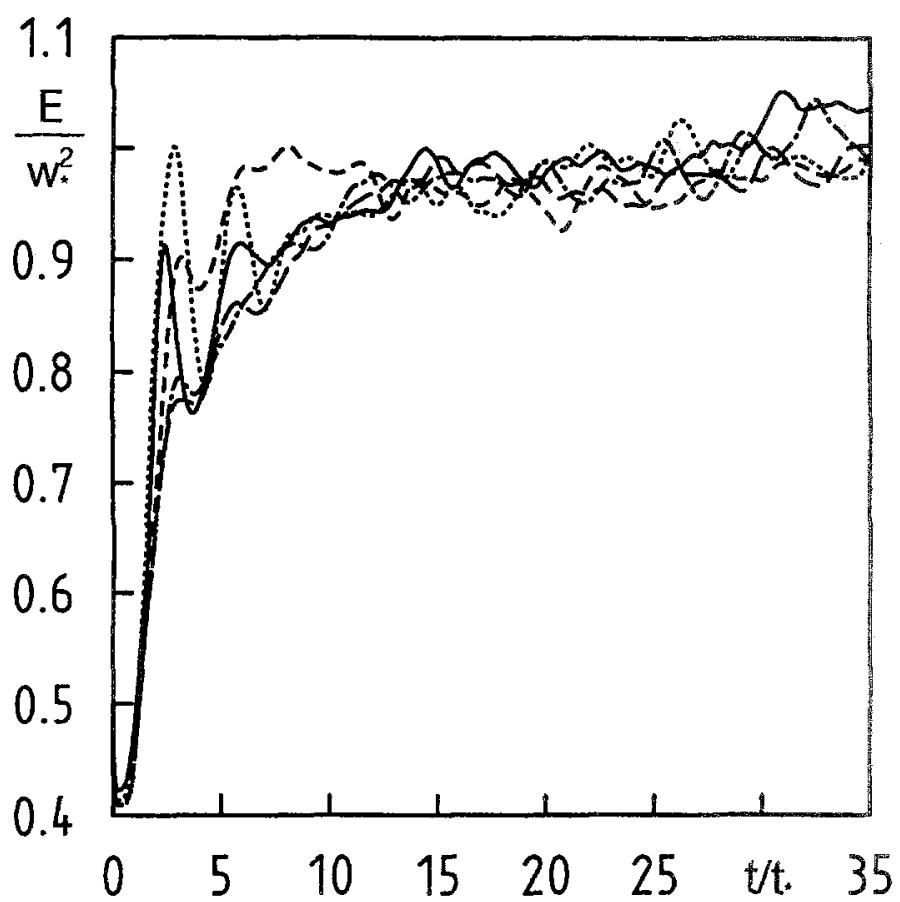

Fig. 2. Spatially averaged kinetic energy versus time $t$ for cases with $U=w_{*}, L=8 H, \delta=0.1 H$, and for various wavelengths, $\lambda / H=$ $1, \ldots-2, \ldots-4, \ldots \cdot 8, \ldots+\infty$

computations until a final time of $35 t_{*}$. This is a rather long time. It corresponds to about $6 \mathrm{~h}$ for a CBL of $1000 \mathrm{~m}$ thickness and a temperature flux $Q_{s}=$ $0.1 \mathrm{~K} \mathrm{~m} \mathrm{~s}^{-1}$ (heat flux of about $100 \mathrm{~W} \mathrm{~m}^{-2}$ ). For flat and homogeneous surfaces, the CBL becomes steady after about 6 convective time units (Schmidt and Schumann, 1989). Hence we expect that our final results are close to steady state.

\section{Results for a Weak Mean Wind}

We shall report results from several cases for various wavelengths $\lambda$ between $H$ and $8 H$. All other parameters are fixed: mean velocity $U=w_{*}$, surface wave amplitude $\delta=0.1 \mathrm{H}$, surface roughness height $z_{0}=10^{-4} \mathrm{H}$, lateral domain size $L=$ $8 H$, number of grid points $128 \cdot 128 \cdot 16$, corresponding to $\Delta x=\Delta z=H / 16$, and time step $\Delta t=0.005 t_{*}$. The selected values allow us to compare our results with those of Krettenauer and Schumann (1992). The influence of other parameter values is discussed in Section 4.

\subsection{Flow structure and approach to steady state}

Figure 2 shows the temporal development of the total kinetic energy of the motions averaged over the total computational domain for five cases with different 
wavelengths. The energy starts from values slightly larger than $U_{b}^{2} / 2=0.408 w_{*}^{2}$ because of initial adjustment of the field according to continuity and the terrain shape in the form of potential flow and very small contributions from the random perturbations in the initial conditions. At first, the energy increases and reaches rather large values due to roll circulations induced by buoyancy under the influence of the wavy surface and the shear. Such regular structures are formed most strongly for $\lambda / H=2$. Later, however, the flow structure becomes more random with increased dissipation, and the energy tends to an asymptotic level at times after $t=$ $15 t_{*}$ for all cases. We observe that even this integral quantity does not reach a strictly steady state. Instead it fluctuates by up to $5 \%$ around an apparently existing asymptotic value at time scales of the order of a few convective time units. The same behaviour was found for zero mean wind by Krettenauer and Schumann (1992). Such fluctuations are to be expected because the domain contains only a finite number of large-scale motion modes so that the volume average differs from a true ensemble mean value. Late in the simulations, there remains a small but finite trend of increasing energy which might be caused by slowly growing horizontal scales of the most energetic motion components with reduced dissipation. In view of the variability of boundary conditions, such long-term trends are irrelevant to atmospheric conditions.

Figures 3 and 4 display the temporal development of the flow structures for two different topographic wavelengths. Contours of constant vertical velocity are plotted in the lower part and at midlevel of the boundary layer in horizontal planes at constant value of the vertical coordinate $z / H$. These results are obtained by interpolating vertically between the velocities at neighbouring $\eta$-levels. The selected plots can be compared with earlier results for flat or wavy surfaces. Moreover, plots of vertical velocity have been found to show the structures most clearly. The pictures obviously exhibit regular coherent structures which carry a large portion of the total energy together with less regular small-scale turbulence. At early times, the upward motion prefers rolls parallel and perpendicular to the wave crests. Later, these structures grow horizontally and become deformed by the mean wind and shear. Basically, the convective structure is similar to that observed over flat surfaces (Schmidt and Schumann, 1989). The upward motions are concentrated into polygonal lines which are rather narrow and enclose large areas of downward motions. However, in contrast to cases for zero mean wind, the polygonal lines of upward motion are no longer closed rings but become disrupted for positive mean wind, mainly on the downstream side. In the middle of the mixed layer, the regions of upward motion merge to form isolated plumes of growing diameter which rise up to the top boundary. The plumes are surrounded by slowly sinking fluid. They stay close to vertical for this wind (and also for stronger winds with $U / w_{*} \leqslant 5$ ). The simulations reveal a trend to form a largescale drift in one direction near the bottom, and in the opposite direction near the top of the layer, as observed experimentally for turbulent convection without mean wind over flat surfaces by Krishnamurti and Howard (1981). 


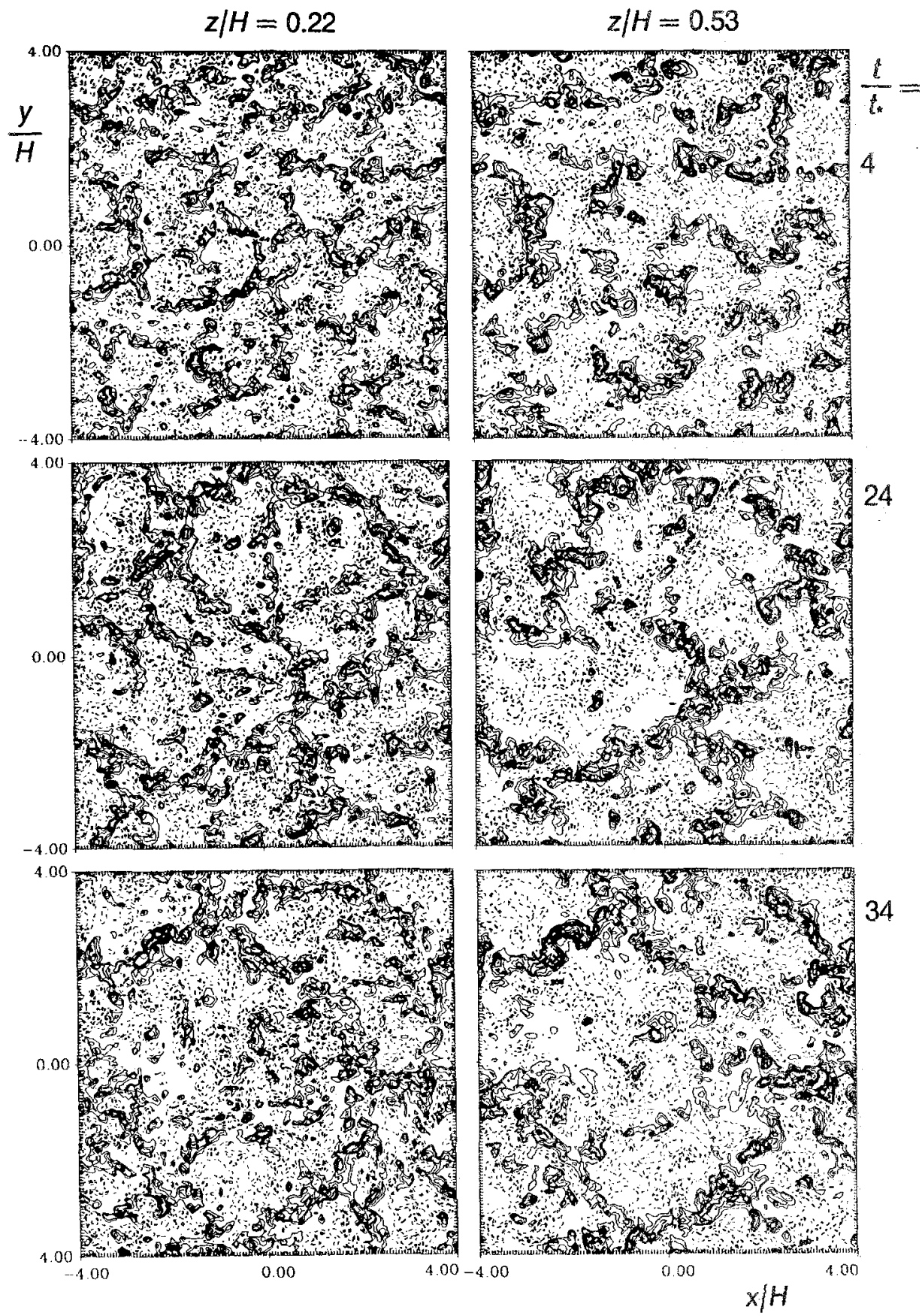

Fig. 3. Contour plot of vertical velocity $w$ for $\lambda / H=1$, in horizontai planes at $z / H=0.22$ (left) and 0.53 (right panels), at $t / t_{*}=4$ (top), 24 (middle), and 34 (bottom). Solid contours for non-negative values at increments $\Delta w^{+}=0.3 w_{*}$, and dashed contours for negative values at increments $\Delta w^{-}=0.4 w$, $U / w_{*}=1, z_{0} / H=10^{-4}, \delta / H=0.1$. 
$z / H=0.22$
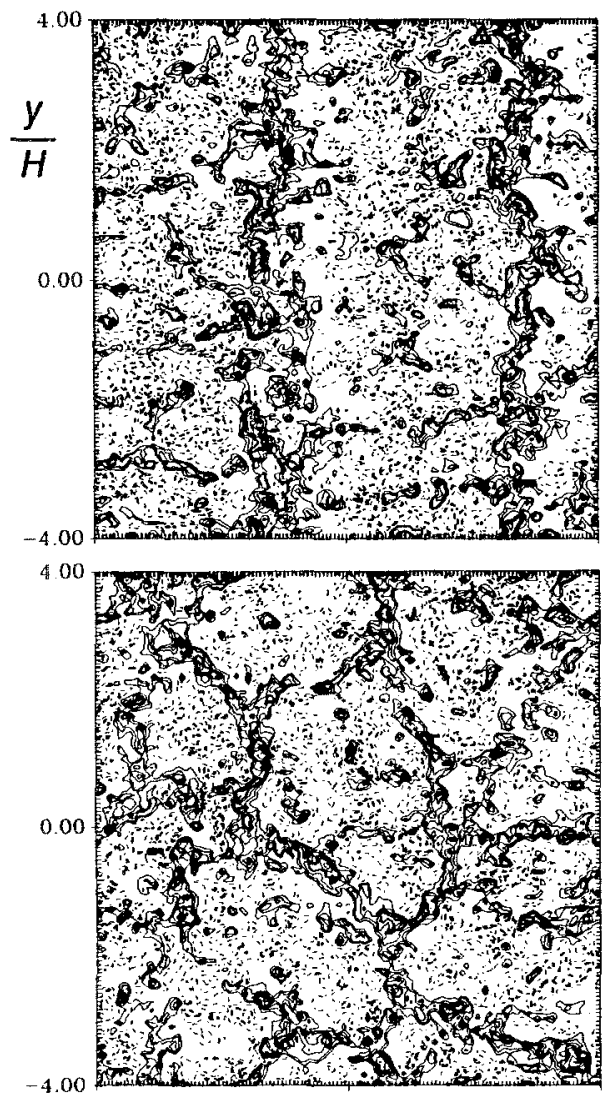
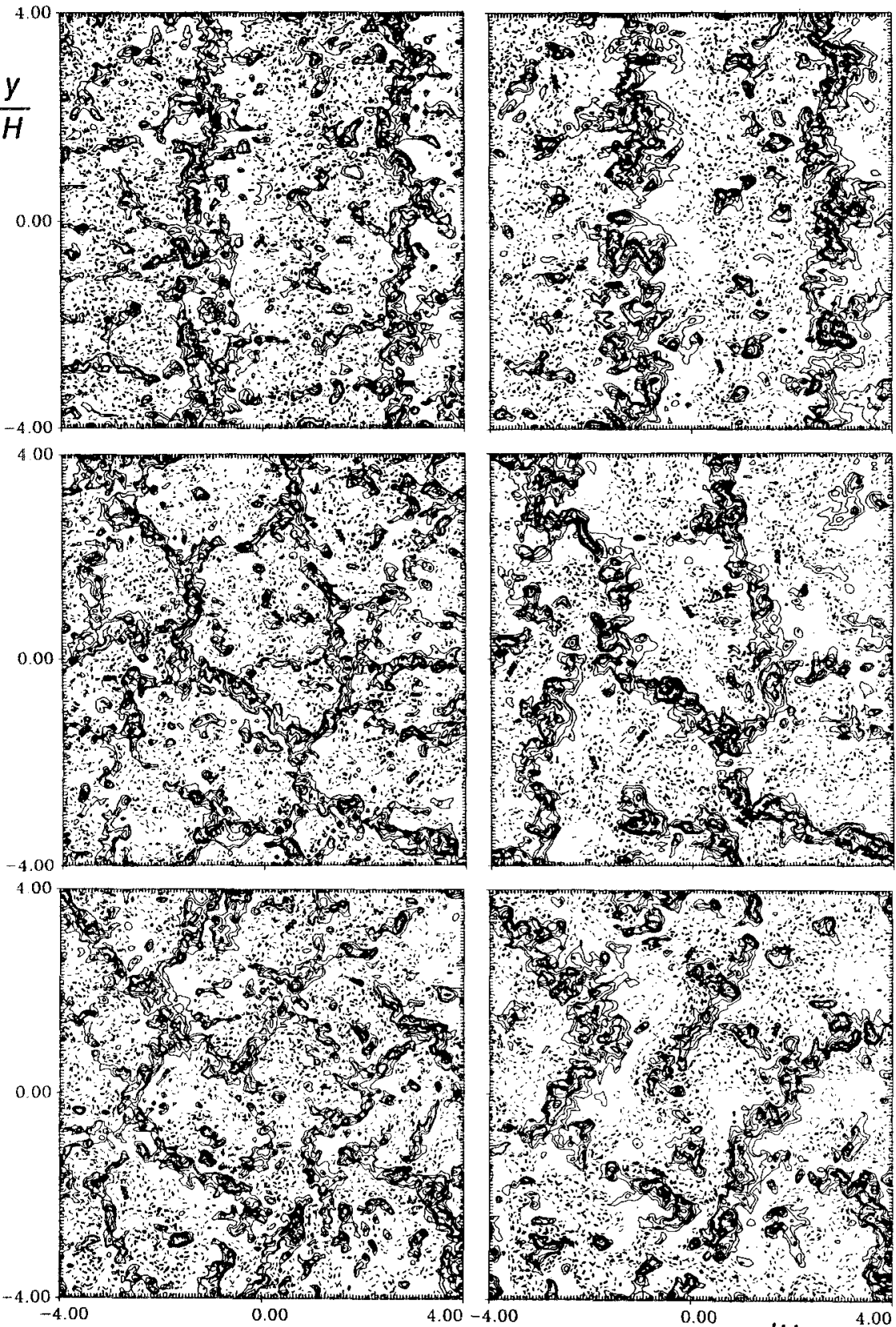

$$
z / H=0.53
$$
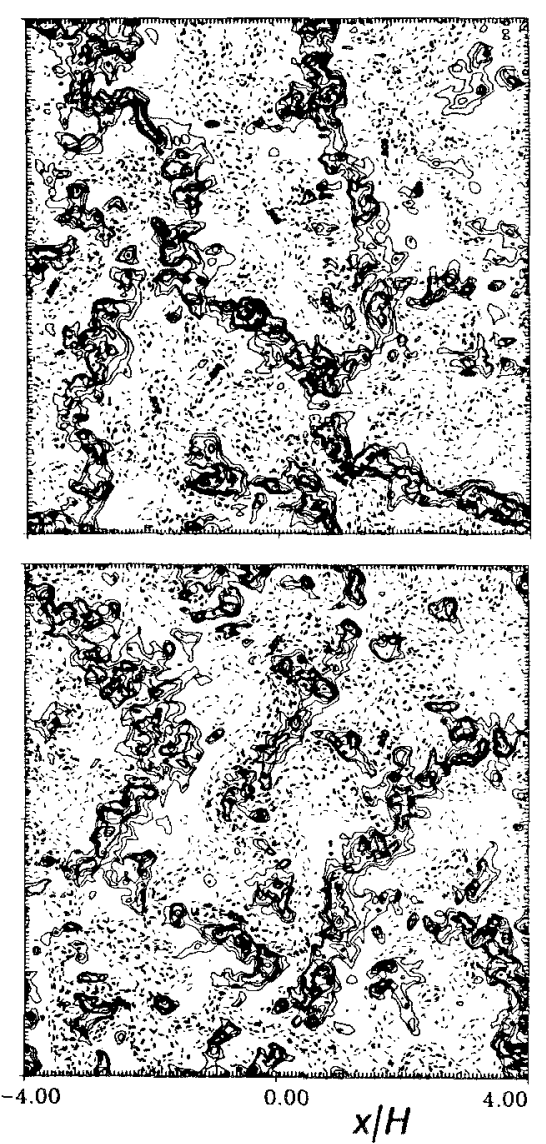

Fig. 4. Same as Figure 3 for $\lambda / H=4$. 
Figure 3 indicates that the motion pattern exhibits only a weak impact from the surface wave in that updrafts form preferentially above wave crests. The weak correlation between upward motion and surface height corresponds to the findings of Krettenauer and Schumann (1992) for zero mean wind. Comparing the results at various times, we find that the horizontal scale of turbulence increases considerably from $t / t_{*}=4$ to 24 , but does not increase significantly thereafter. Obviously, the motion scales are not limited by the size of the domain in a noticable manner. From these and further pictures at various times and levels we found that the polygonal lines are preferentially oriented along the diagonal in $x-y$-planes. This is most obvious for $\lambda / H=2$ and might indicate a superposition of rolls in the downstream and cross-stream directions which are triggered by the surface waves. However, these directional orientations are only weak.

Figure 4 shows that the surface has a stronger impact on the motion structure for $\lambda / H=4$ than for $\lambda / H=1$. The differences are large at early times but decrease at late times. By inspection of similar pictures for larger wavelengths, it was found that the turbulence structure is most sensitive to the surface form (at this low wind speed) for $\lambda / H=4$. This wavelength is also the most sensitive one for zero wind speed (Schumann, 1991b). The figures show again only small structural changes after $t / t_{*}=24$, and are therefore representative of a steady state.

\subsection{MEAN PROFILES}

In this section we investigate the influence of the surface undulation on the turbulent motion in terms of mean profiles, which are averages at constant vertical coordinate $z$ unless stated otherwise. For heights $z<\delta$, the averages are mean values over the fluid part only. The results show systematic differences to corresponding ones at constant $\eta$ because of strong horizontal variations of the variances at low altitudes. The profiles vary smoothly with $\eta$ but show stronger changes at $z=\delta$ when averaged at constant altitude.

Figure 5 shows the mean velocity profile normalized by the bulk mean velocity $U_{b}$ at an early time and at a late time of the simulations. In the transient initial period, at $t=4 t_{*}$, the short-wave cases are strongly sheared across the height of the CBL. This suggests a strong resistance at the wavy surface, which decreases with wavelength. Later, the CBL becomes well mixed so that the velocity becomes uniform for all altitudes above $0.2 H$. The remaining shear near the surface is strongest for the shortest wavelength. For large wavelength, the variations are within the statistical uncertainty of such averages. The mean velocity is strongly reduced below the crests of the surface waves but remains positive throughoui, without recirculation in the valleys.

As shown in Figure 6, the temperature profile reaches a steady shape much earlier. The increase from $t=4 t_{*}$ to $t=24 t_{*}$ amounts to 20 units in $T_{*}$ because the layer is heated from below at a corresponding rate. The mixed layer is unstable in the lower half because of surface heating. In spite of the heating from below, the mean temperature in the upper part of the layer increases with height. This 

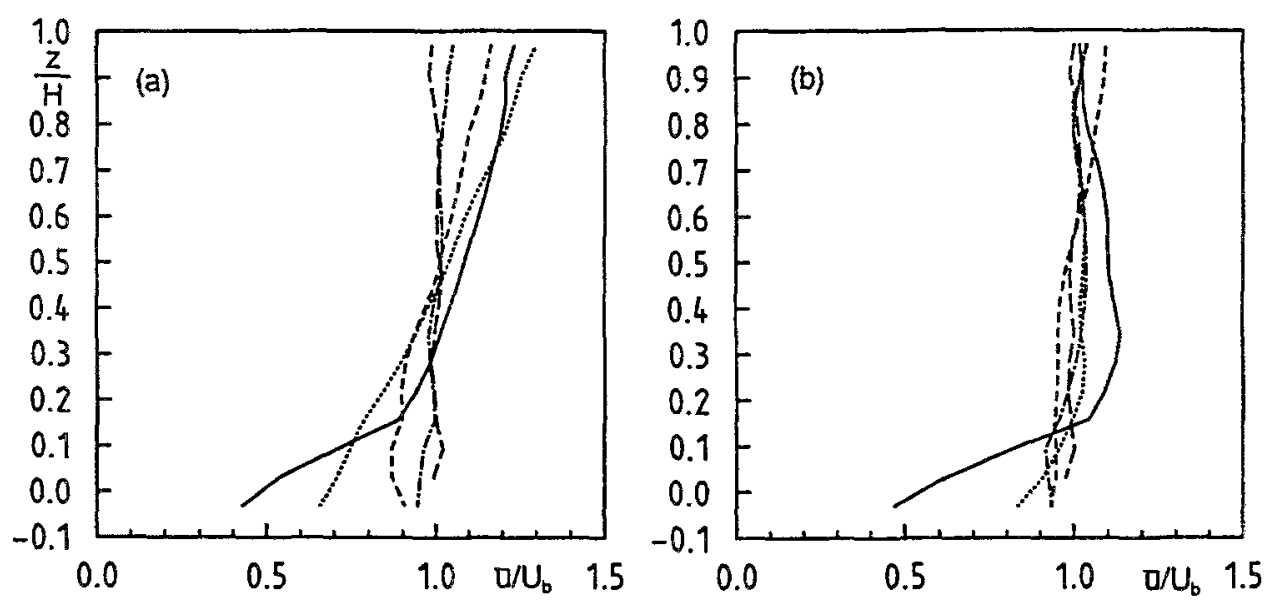

Fig. 5. Mean velocity profile $\bar{u} / U_{b}$ versus vertical coordinate $z / H$, for various wavelengths; parameters and line-coding as in Figure 2. (a) $t / t_{*}=4$, (b) $t / t_{*}=24$.

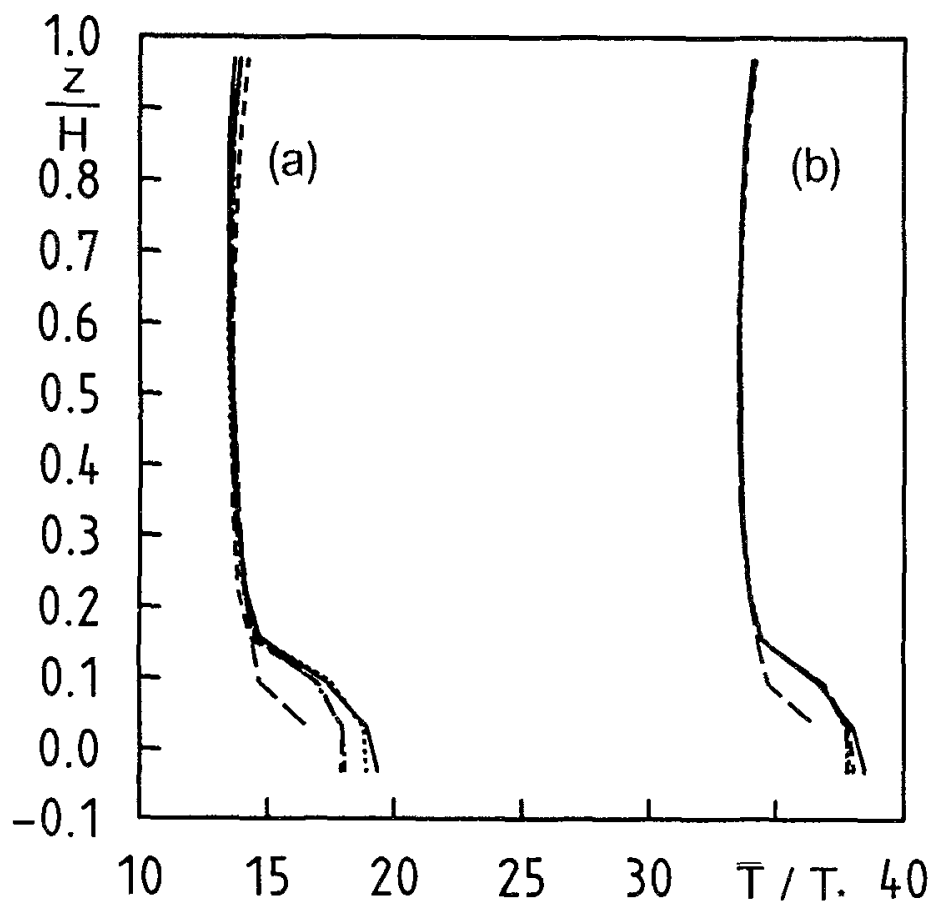

Fig. 6. Mean temperature profile $\bar{T} / T_{*}$ versus $z / H$ at (a) $t / t_{*}=4$, and (b) $t / t_{*}=24$. Parameters and line coding as in Figure 2. 

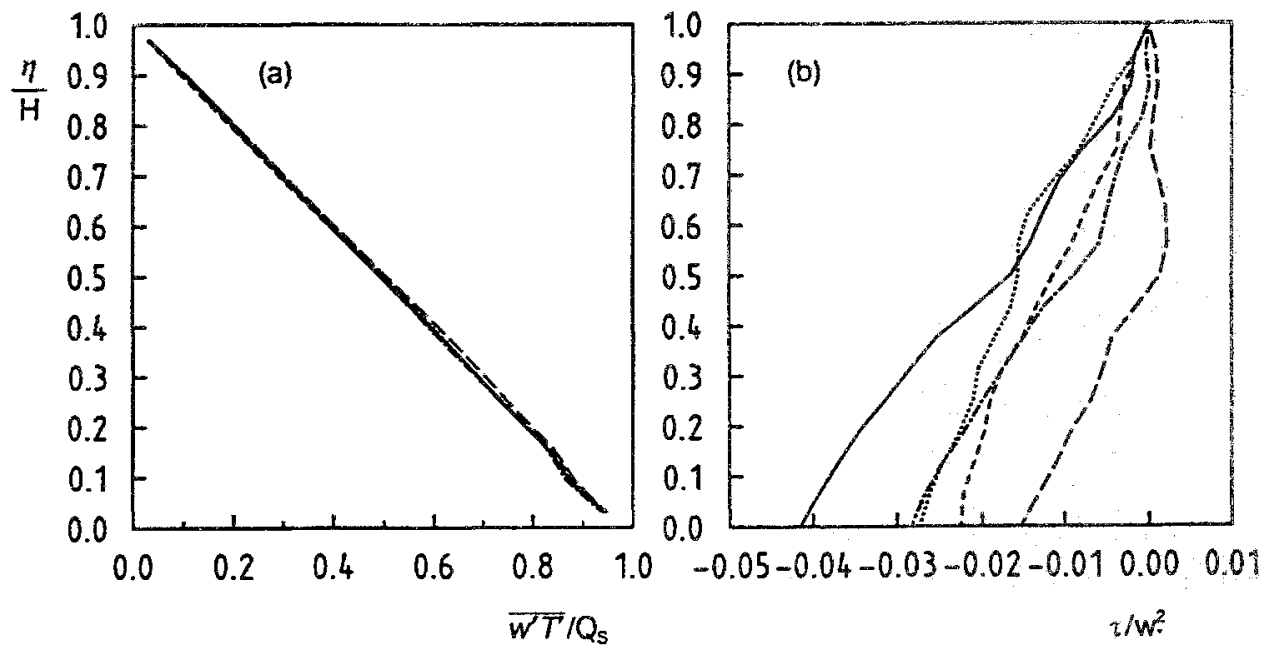

Fig. 7. (a) Vertical heat flux profile $\overline{w^{\prime} T^{\prime}} / Q_{S}$ versus $\eta / H$ averaged within the interval $28.5 \leqslant c / t_{*}$ $\leqslant 33.5$. (b) Same for vertical momentum flux $\overline{u^{\prime} w^{\prime}} / w_{*}^{2}$. Parameters and line coding for various wavelengths as in Figure 2.

implies a countergradient heat flux, caused by narrow and tast rising clements transporting heat from the lower surface directly up to the upper part of the fluid layer; the remainder of the fluid within the mixed layer is heated from above by turbulent diffusion and sinking warm fluid. The countergradient transport has also been observed for zero mean wind by Krettenauer and Schumann $(1989,1992)$ and Walko et al. (1992). Above the minimum at midlevel, the temperature increases by about $0.7 T_{*}$ corresponding to a Brunt-Väisälä frequency $N \cong 1.2 t_{*}^{-1}$. This stable stratification allows for slow gravity oscillations with periods of the order $2 \pi / N \cong 5.3 t_{*}$. The related period of kinetic energy is half that value and might explain some of the oscillations in Figure 2. In the valleys, the fluid moves slower and therefore gets warmer than over flat surfaces by typically $2.5 T_{*}$.

The vertical fluxes of heat and momentum are averaged at constant values of the coordinate $\eta$; see (1). The vertical heat flux is shown in Figure 7a for a late time. The kink at the lowest grid point is due to differences between computation of the advective fluxes in the integrations (by a second-order upwind scheme) and analysis of the statistics (using centered averages) and is irrelevant, therefore. It was found that the expected linear profile, which is required for steady state, is reached most slowly for $\lambda / H=2$ and 4 . This indicates that these wavelengths are most selective to large-scale structures which reach a steady state slowly. The final result shows a very small departure from linearity.

The mean vertical flux of downstream momentum per unit volume at constant height $z$ is $\overline{u^{\prime} w^{\prime}}$. Both the resolved motions of the large eddies and the modelled fluxes of the SGS motions contribute to this flux. At undulated surfaces; however, pressure forces cause an additional momentum flux. Therefore, we compute the 
mean vertical momentum flux per unit volume $\tau$ at constant coordinate $\eta$; see Figure $7 \mathrm{~b}$, which is the sum of the resolved flux $\tau_{\text {res }}$, SGS or frictional contribution $\tau_{\text {fric }}$, and pressure contribution $\tau_{\text {pres. }}$. The latter is computed from

$$
\tau_{\text {pres }}=-\rho^{-1} \overline{p(\partial z / \partial x)_{\eta}} .
$$

where $\rho$ is the mean fluid density and the bar denotes the average over coordinate planes with $\eta=$ const. The pressure is partly induced by hydrostatic forces due to temperature variations within the fluid and partly by dynamic forces due to pressure head and friction. The SGS flux is small inside the flow domain but significant at the surface. At flat surfaces, it forms the only non-zero contribution corresponding to the friction drag. Its magnitude depends on the surface roughness which enters the Monin-Obukhov conditions used at the surface. The momentum flux profiles deviate from a linear profile much more than the heat flux profiles. This difference is caused by the different processes which are responsible for fluxes of heat and momentum. The heat flux is carried by smaller scale motions than the momentum flux so that the number of independent events is smaller for momentum than for heat transport; this causes smaller statistical fluctuations for the latter. In fact, near the surface, the heat flux is carried by eddies which scale with the height above the surface whereas the momentum flux is controlled to a large extent by pressure forces which scale with $\lambda$. Also, gravity waves in the upper part of the mixed layer may contribute to oscillations in the momentum flux but will affect the heat flux to a lesser extent. However, the total momentum flux, Figure $7 \mathrm{~b}$, is sufficiently close to the expected linear shape that the results can be taken as being quasi-steady.

From measurements of the spectra of turbulence in the CBL, Kaimal et al. (1982) showed that the lengthscales of vertical velocity fluctuations are larger and the scales of temperature fluctuations are smaller over rugged terrain than over smooth terrain. These findings are consistent with our interpretation of the flux oscillations.

The contributions from friction and pressure drag and the total drag are listed in Table I. For the shortest wavelength, the frictional drag is $18 \%$ smaller than over flat surfaces, presumably because of the large areas with reduced flow speed in the valleys. The pressure drag at the surface has been split into its dynamic and its hydrostatic parts,

$$
\tau_{\text {pres }}=\tau_{\text {dyn }}+\tau_{\text {hydr }},
$$

The hydrostatic pressure part is evaluated from Equation (3) for $z=h(x)$ using

$$
p_{\text {hydr }}=-\int_{h}^{H} \rho \beta g\left(T-T_{b}\right) \mathrm{d} z,
$$

where $T_{b}$ is the bulk mean temperature. This pressure measures the weight of the fluid column above the surface as induced by density differences due to variable 
TABLE I

Surface fluxes and temperature difference as a function of external parameters

\begin{tabular}{lllllcccc}
\hline$\lambda / H$ & $\delta / H$ & $U_{b} / w_{*}$ & $\tilde{u}_{*} / w_{*}$ & $\tau / w_{*}^{2}$ & $\tau_{\mathrm{fric}} / w_{*}^{2}$ & $\tau_{\mathrm{dyn}} / w_{*}^{2}$ & $\tau_{\mathrm{hydr}} / w_{*}^{2}$ & $\Delta T / T_{*}$ \\
\hline$\infty$ & 0 & 0 & 0.113 & 0 & 0 & 0 & 0 & 49.1 \\
1 & 0.1 & 0 & 0.123 & 0 & 0 & 0 & 0 & 49.8 \\
1 & 0.1 & 0.9 & 0.142 & -0.0416 & -0.0126 & -0.0106 & -0.0184 & 48.9 \\
1 & 0.1 & 1.8 & 0.186 & -0.0732 & -0.0310 & -0.0289 & -0.0133 & 46.7 \\
1 & 0.1 & 4.5 & 0.334 & -0.1914 & -0.1122 & -0.0743 & -0.0050 & 37.1 \\
1 & 0.15 & 0.85 & 0.138 & -0.0965 & -0.0089 & -0.0460 & -0.0417 & 50.1 \\
2 & 0.1 & 0.9 & 0.138 & -0.0275 & -0.0137 & -0.0005 & -0.0132 & 48.6 \\
4 & 0.1 & 0.9 & 0.141 & -0.0226 & -0.0157 & -0.0001 & -0.0068 & 48.3 \\
8 & 0.1 & 0.9 & 0.141 & -0.0284 & -0.0148 & +0.0095 & -0.0231 & 48.1 \\
$\infty$ & 0 & 0.9 & 0.139 & -0.0154 & -0.0154 & 0 & 0 & 48.3 \\
\hline
\end{tabular}

In Table I, $\tilde{u}_{*}$ is the root-mean-square friction velocity at the surface (computed locally from the vector sum of the frictional fluxes in the $x$ and $y$-directions); $\tau$ is the total vertical flux of downstream momentum per unit volume at the surface; $\tau_{\mathrm{fric}}, \tau_{\mathrm{dyn}}$, and $\tau_{\mathrm{hydr}}$ are the corresponding flux components due to friction forces, dynamic pressure forces, and hydrostatic pressure forces, respectively; $\Delta T$ is the averaged temperature difference between the surface (at roughness height) and the bulk mean temperature. The results are averages over the bottom surface and over the time interval from 28.5 to $33.5 t_{*}$.

temperature. The dynamic pressure part is computed from the difference between the total and the hydrostatic pressure part. The pressure drag components are largest for the shortest wavelength and vanish for flat terrain. We find a nonmonotonic variation with wavelength (see Table I). The total pressure drag $-\tau_{\text {pres }}$ reaches a local minimum for $\lambda / H=4$, and the dynamic part $-\tau_{\text {dyn }}$ is slightly negative for $\lambda / H=8$. This indicates changes in flow dynamics as will be discussed below. For all these cases with $U=w_{*}$, the friction velocity $u_{*}=(-\tau)^{1 / 2}$ (based on the total momentum flux at the surface) is small in the sense that $u_{*} / w_{*}<0.2$. Hence, the shear is too weak to drive coherent longitudinal roll motions, according to the criterion of Sykes and Henn (1989). However, in agreement with Brutsaert and Kustas (1987), the shear forcing is much larger over the wavy surface than over flat surfaces.

The turbulence intensity is measured by the variances of the velocity and the temperature fluctuations, see Figure 8 . The downstream velocity variance is large near the surface and even larger near the top surface with a minimum in the midlayer. This variance pattern suggests that part of the turbulent motions are organized into strong coherent rolls with axes parallel to the wave crests experiencing stronger friction and turbulent destruction at the lower surface than at the frictionless top boundary. From plots at various times, we found that this variance reaches a steady state at about $t=14 t_{*}$. Initially, the variance grows most quickly for $\lambda=4 H$, followed by $\lambda=2 H$ whereas the profiles for other (shorter and larger) wavelengths stay more closely together at all times. At late times, the variance is smallest for $\lambda$ between 2 and $4 H$. This indicates again some special dynamics at the intermediate range of wavelengths. The differences between the profiles for 

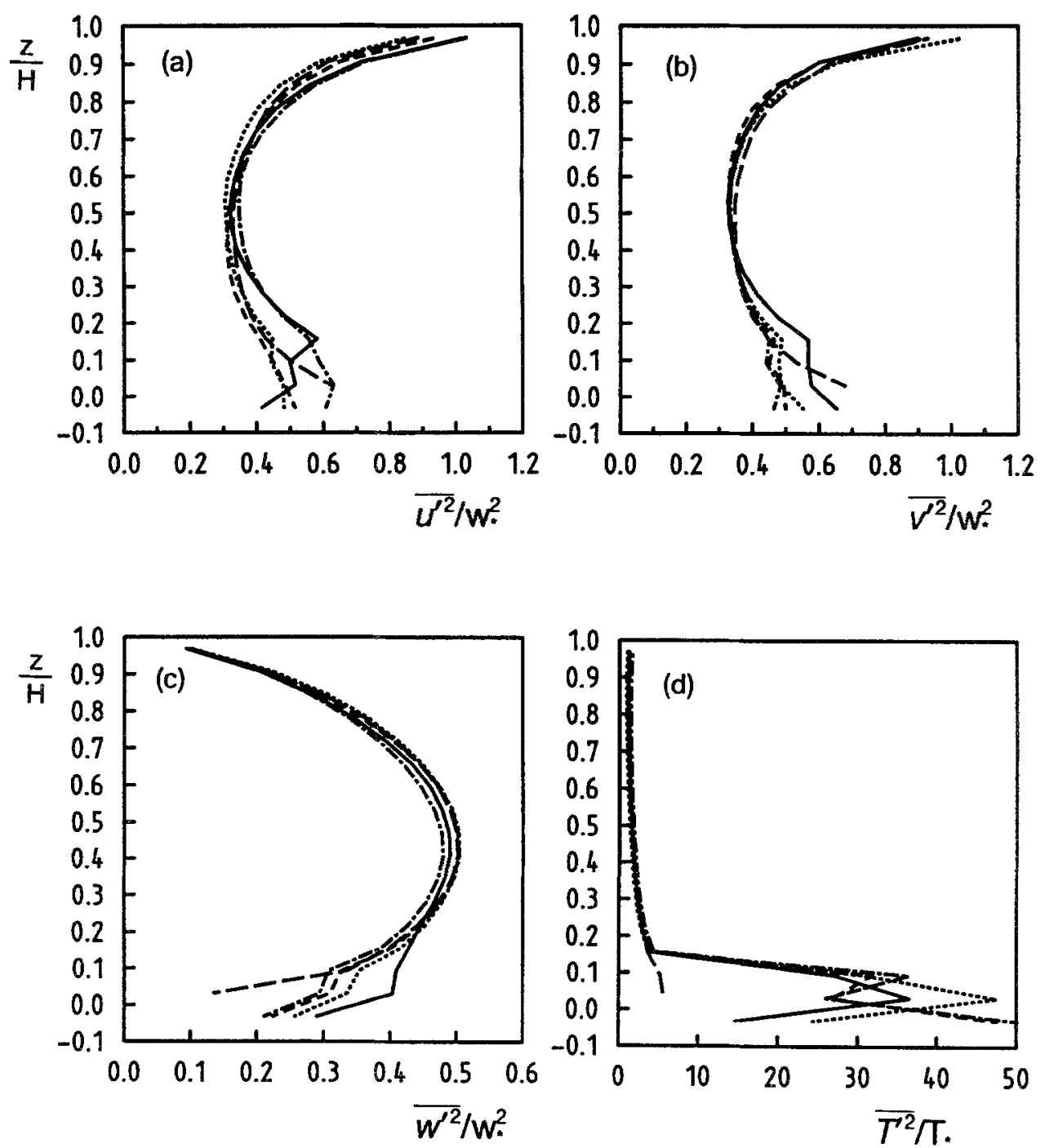

Fig. 8. Vertical profiles of turbulence variances versus $z / H$ averaged in the time interval $28.5 \leqslant t / t_{*}$ $<33.5$. Parameters and line coding as in Figure 2. (a) downstream velocity $\overline{u^{\prime 2}}$, (b) cross-stream

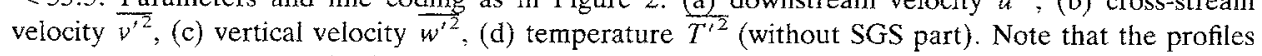
are averages over the flow domain at fixed height, not at fixed transformed coordinates.

various wavelengths is smaller than for zero mean wind (Figure 11 of Krettenauer and Schumann, 1992). Hence, coherent roll motions with axes parallel to the crests, which are important at zero mean wind, are less pronounced in this case with weak mean wind. In the valleys, for steep surface waves, the velocity fluctuations are larger than the small positive mean velocity, so that the fluid recirculates in part of the time. Figure $8 \mathrm{~b}$ shows the corresponding results for the cross-stream 
variance, which is very similar to the downstream variance in shape and magnitude. This reveals roll motions also with axes perpendicular to the wave crests. The lateral variance near the surface increases by about $10 \%$ from time 28.5 to $33.5 t_{*}$, which appears to be the strongest indication that the trend to form longitudinal rolls has not yet come to an end. The vertical velocity variance is much closer to steady state. Figure $8 \mathrm{c}$ shows the profile at a late time. It is affected by the terrain only for $z / H \leqslant 0.2$, where strong kinematical contributions are to be expected. In the mixed layer, $\overline{w^{\prime 2}}$ reaches a maximum value of about 0.47 to $0.5 w_{*}^{2}$. This value is the same as for zero mean wind speed (see Figure 11 of Krettenauer and Schumann, 1992).

The temperature variance (without SGS contribution), see Figure $8 \mathrm{~d}$, is largest near the surface, as to be expected because that is the region with maximum values of the negative product of heat flux and temperature gradient, which is responsible for generation of such fluctuations. Above the wave crests, the absolute values are close to those for flat terrain and zero mean wind. However, inside the valleys the temperature fluctuations are much larger. The local minimum of the temperature variances near $z=0$ for $\lambda \geqslant 4 H$ indicates a change in flow pattern as will be discussed below. Since the vertical scale of temperature fluctuations is small near the surface, the large magnitude of these fluctuations near the surface causes the stratification in the valleys to oscillate between stable and unstable conditions.

\subsection{Phase-averaged flow patterns}

Figures 9 and 10 show the phase-averaged horizontal and vertical velocity fields in vertical planes for most of the cases considered in this study. Panels c to f show the results for different wavelengths. The results for other parameter values (panels $\mathrm{a}, \mathrm{b}$, and g) will be discussed later. The fields represent averages over the lateral coordinate, time and over positions of equal phase angle relative to the surface wave.

Note that this average filters out all horizontal scales longer than $\lambda$, which carry a large fraction of energy. For this reason, for short topographic wavelengths $\lambda$, the phase-averaged flow looks very smooth and similar to a potential flow although the instantaneous motion structure is much different from those of irrotational flows. The mean flow over the surface kinematically enforces strong upward and downward motion at the slopes. The horizontal velocity is larger than average over the crests of the surface waves. Just above the crests we observe an overshoot in velocity which is typically of order 10 to $15 \%$ of $U$. Certainly, the details of the vertical profile of the horizontal velocity above the crest are only crudely resolved by the rather coarse numerical grid. Perturbations in a potential flow above a sinusoidal surface of horizontal wavelength $\lambda$ decay on a vertical scale $\lambda /(2 \pi)$ (Mason, 1987). We observe, in fact, that the disturbances induced by the surface wave are small above that height. In the valleys, the downstream velocity is strongly reduced, indicating departure from potential flow with strong vertical 


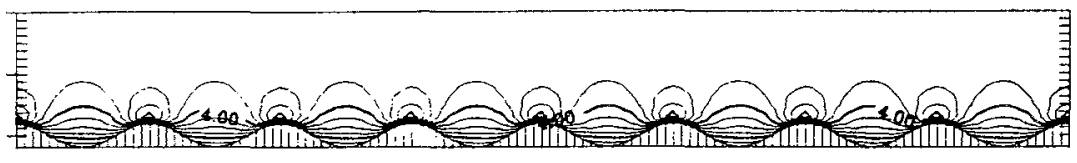

(a)
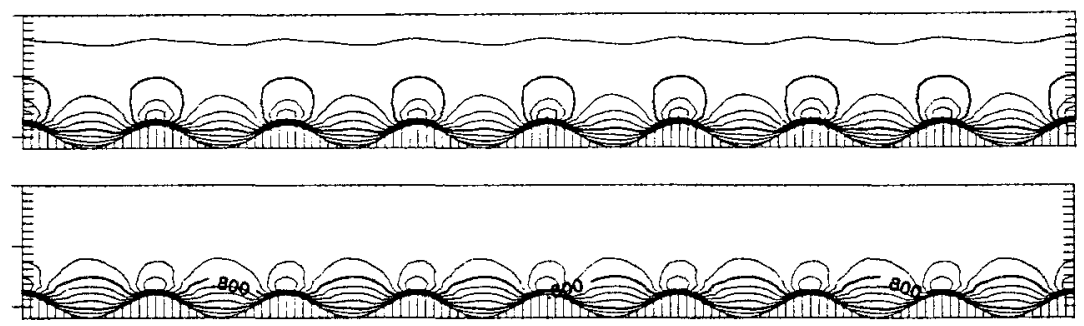

(c)
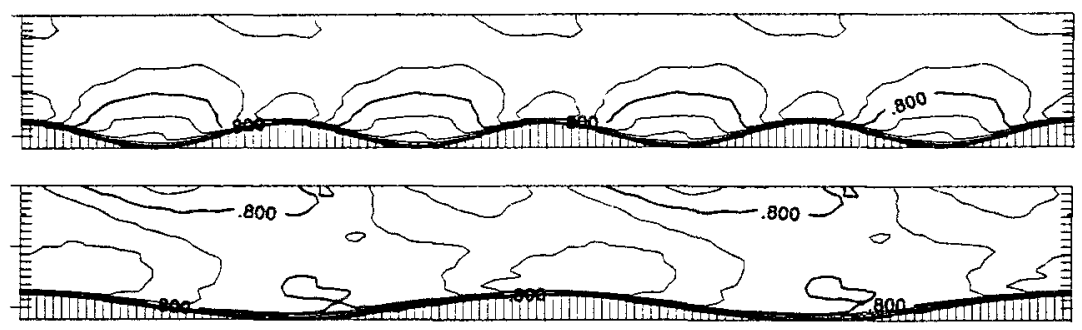

(e)
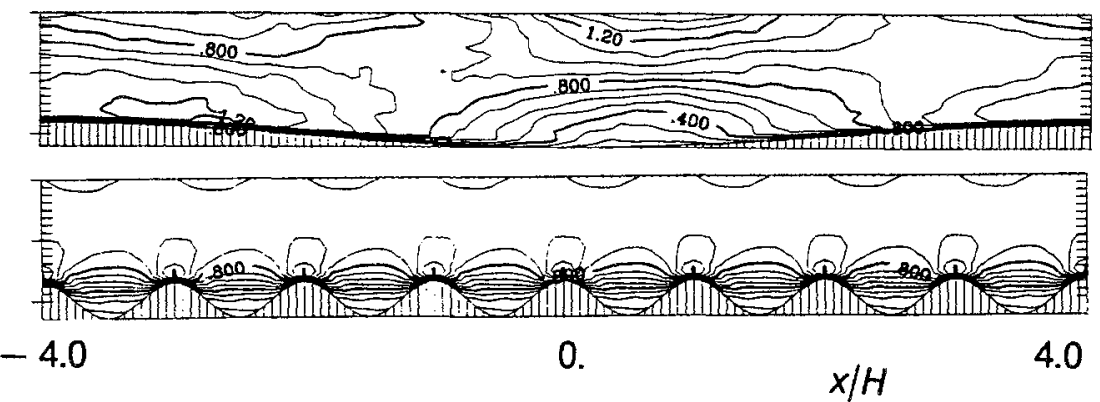

$(g)$

Fig. 9. Phase-averaged horizontal velocity $u$ in a vertical plane for various values of $U / w_{*}, \lambda / H, \delta / H$, and contour increments $\Delta u / w_{*}$, respectively: (a) $5,1,0.1,0.5$, (b) $2,1,0.1,0.2$, (c) $1,1,0.1,0.1$, (d) $1,2,0.1,0.1$, (e) $1,4,0.1,0.1$, (f) $1,8,0.1,0.1$, (g) $1,1,0.15,0.1$.

mixing. The downstream velocity stays positive in all cases, so that recirculation zones are absent. For larger wavelengths, the flow shape departs more strongly from what one would expect kinematically. Narrow updrafts are formed extending over the whole boundary layer. However, these phase-averaged updrafts have rather small vertical velocities less than $0.17 w_{*}$. The position of the updraft forming at the upslope side moves backwards for longer waves. For $\lambda=4 H$, the updraft rises from the beginning of the upslope side and for $\lambda=8 H$, the updraft is strongest already at the end of the downslope before the floor of the valley. For $\lambda=8 \mathrm{H}$, the horizontal velocity exhibits a strong minimum after the updraft. The updraft transports warm fluid from the valley floor to the top of the mixed layer and cooler 

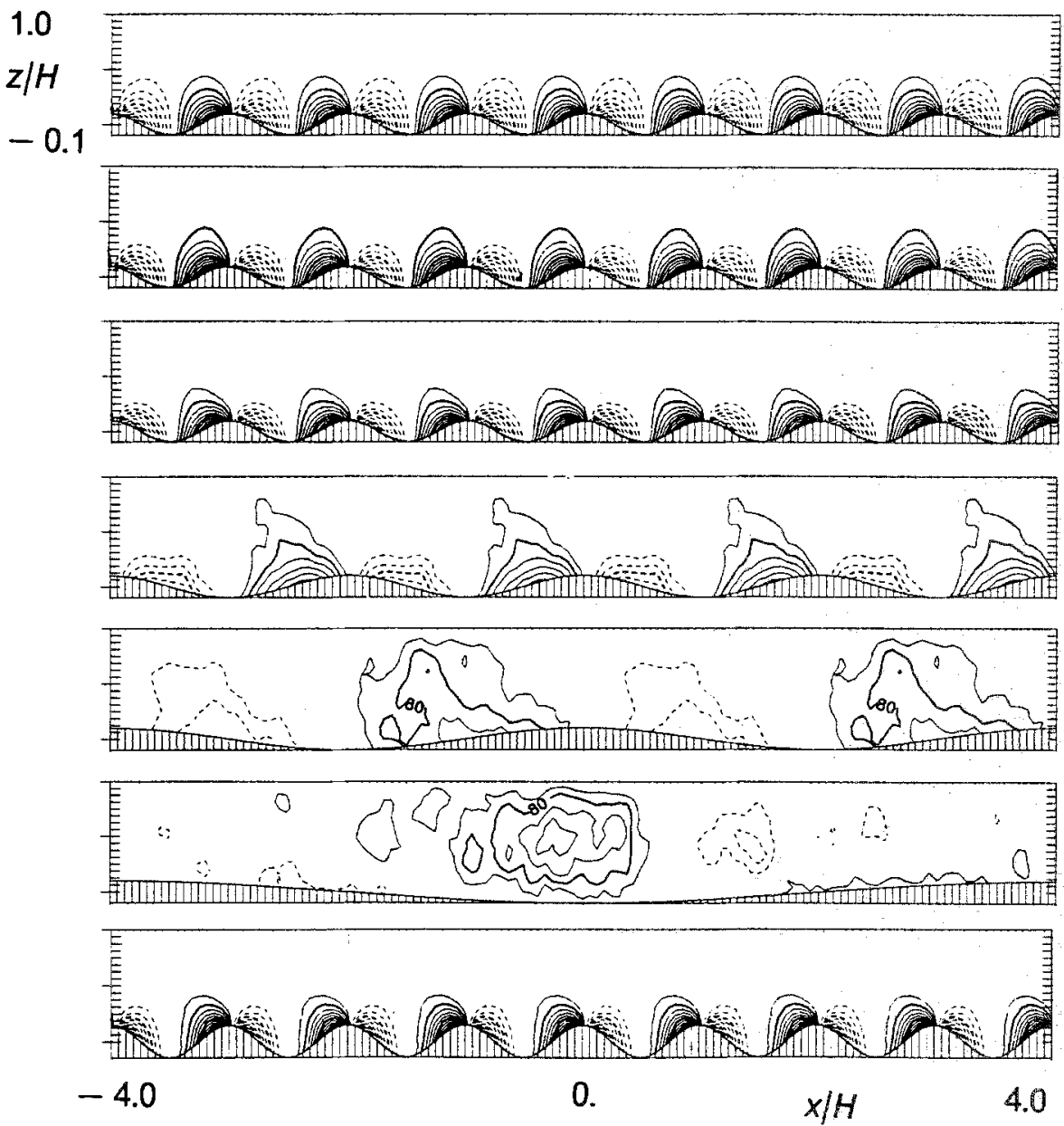

Fig. 10. Same as Figure 9 for vertical velocity $w$. The contour increments are (a): 0.2, (b): 0.08 , (c) to $(\mathrm{g}): 0.04$

air from above is transported to the upslope side by lateral motions around the updraft. Behind the updraft, in the slowly moving fluid, the temperature increases more strongly so that the fluid becomes relatively warm at the upslope.

For short waves, the fluid column above the upslope is relatively cold so that the surface pressure is large there. Correspondingly, the downslope side experiences small hydrostatic pressure. This contributes to a negative value of the pressure drag $\tau_{\text {pres }}=-\rho^{-1} p \partial h / \partial x$. In this sense, pressure drag for buoyant flows is of a different nature than form drag for stronger flows where dynamic pressure forces dominate. The wavelength $\lambda=4 H$ is special in the sense that it is close to the inherent scale of turbulent convection, which explains the non-monotonic decrease in pressure drag for increasing wavelength; see Table I. 


\section{TABLE II}

Impact of domain size $L$, horizontal grid spacing $\Delta x$ and vertical grid spacing $\Delta z$ on surface fluxes for the reference case with $\lambda / H=1, U / w_{*}=1, \delta / H=0.1, z_{0} / H=10^{-4}$.

The last row gives the percentage differences between the third and the fifth case

\begin{tabular}{llllllll}
\hline$L / H$ & $\Delta x / H$ & $\Delta z / H$ & $\tilde{u}_{*} / w_{*}$ & $-\Delta T / T_{*}$ & $\tau_{\text {f ric }} / w_{*}^{2}$ & $\tau_{\text {pres }} / w_{*}^{2}$ & $\tau / w_{*}^{2}$ \\
\hline 8 & $1 / 16$ & $1 / 16$ & 0.142 & 48.9 & -0.0126 & -0.0290 & -0.0416 \\
8 & $1 / 8$ & $1 / 16$ & 0.149 & 48.5 & -0.0144 & -0.0242 & -0.0386 \\
4 & $1 / 16$ & $1 / 16$ & 0.139 & 48.9 & -0.0121 & -0.0303 & -0.0423 \\
4 & $1 / 32$ & $1 / 16$ & 0.132 & 49.5 & -0.0104 & -0.0385 & -0.0489 \\
4 & $1 / 16$ & $1 / 32$ & 0.151 & 47.9 & -0.0129 & -0.0341 & -0.0470 \\
0 & 0 & 100 & 8 & 2 & 6 & 11 & 10 \\
\hline
\end{tabular}

\section{Results for Other Parameter Values}

\subsection{Numerical PaRAMETER STUdies}

In order to test the influence of numerical approximation errors, the reference case with $U / w_{*}=1, \lambda / H=1, \delta / H=0.1, z_{0} / H=10^{-4}, L / H=8$, and $\Delta x=\Delta z=$ $H / 16$, has been run, also with halved and doubled grid spacings, and with halved lateral domain lengths. The smaller grid spacings (and the case with $U=5 w_{*}$ ) require a smaller time step of $\Delta t=0.0025 t_{*}$. From these simulations, the following conclusions can be drawn.

The total kinetic energy, as shown for the reference case in Figure 2, gives almost the same results for $t / t_{*} \leqslant 10$, for all grids and domain sizes. Thereafter, we observe larger fluctuations for the smaller domain size. This supports our conjecture that the final fluctuations are mainly caused by the limited domain size. The simulations with smaller grid spacings give about 5\% larger mean kinetic energy. Because of the additional contributions of the smaller scales, such an increase is to be expected but is unimportant. Inspection of the vertical profile of kinetic energy shows that most of this additional energy arises in the upper $20 \%$ of the domain, whereas the profiles are indistinguishable in the lower part. Variations in the profiles for the variances of the various velocity components and the temperature fluctuations are within the statistical uncertainty of the mean profiles. Hence, the LES results presented are very weakly sensitive to the size of the domain and the horizontal grid spacing.

With respect to vertical grid spacing, the parameter studies show small changes in the statistics of the flow within the mixed layer. As to be expected, the largest changes occur in the surface fluxes. Table II lists the fluxes for various cases with different resolution. Part of the differences in the results is due to statistical errors. However, the surface fluxes increase typically between 6 and $11 \%$ if we reduce the vertical grid spacing $\Delta z / H$ from $1 / 16$ to $1 / 32$. The temperature difference at the surface is less affected by this change since it depends on the square root of the frictional momentum flux at the surface. If the approximation errors scale with the first power of the vertical grid spacing, then a $10 \%$ error for halving the grid 
spacing implies a $20 \%$ error in the limit to very small grid spacings. Certainily, this is not a very reliable estimate but should give about the right magnitude of the approximation errors involved in these simulations.

\subsection{Physical parameter studies}

In this section we consider the reference case and vary either the mean wind or the amplitude of the surface wave.

(a) Mean velocity: $U / w_{*}=0,1,2,5$.

As to be expected, and shown by Sykes and Henn (1989) for flat surfaces, the shear induced by stronger mean winds has a strong impact on the structure and the statistics of the flow if $U / w_{*}$ is large so that $u_{*} / w_{*}$ exceeds about 0.35 . As shown by Figure 11, the structure is fairly invariant to the mean flow for $U / W_{*}$ $\leqslant 2$. However, for stronger mean wind, the surface layer experiences strongly forced upward and downward motions at the upslope and downslope sides of the surface waves. Moreover, the mean shear induces longitudinal rolls with transversal scale of the order $4 H$. This structure is also clearly visible in plots of the lateral velocity $v$ in the surface layer and near the top boundary. Hence these rolls span over the whole boundary-layer. However, it should be noticed that the rolls have narrow (width 0.2 to $0.8 H$ ) upward motion branches with very wide ( 3 to $4 H$ ) downward motion branches inbetween. At $U / w_{*}=2$, a closer inspection of the motion pictures indicates that the polygonal lines of upward motions are oriented systematically by $45 \pm 10^{\circ}$ with respect to the mean flow and the wave crests. This suggests a superposition of longitudinal and transverse rolls at small mean wind speed.

From a computer-generated movie (Raasch und Groß, 1992), showing temperature and particle motions in a vertical plane based on the LES data for the case with $U / w_{*}=2$, we observed a phenomenon of quasi-periodically rising bubbles, which was described by Scorer (1978), p. 344: “. . in the lee of a . . cliff there is a body of slow moving air which gets hotter than the surrounding air because it does not have its heat diffused upwards by turbulence as much as in the case of fast moving air. Therefore from time to time it generates an accumulation of buoyancy which causes it to rise as a thermal." This is exactly what can be observed from the simulations. As sketched in Figure 12, cool stagnant air fills the valley, gets heated and leaves as a thermal sucking cooler air from above and from the sides into the valley. The heating of the air in the valleys is partly a result of lower wind speed, coupled to the lower boundary condition of constant, spatially uniform heat flux, as well as reduced mechanical mixing. This process repeats quasiperiodically. This mechanism destroys any steady recirculation zone.

Figure 13a shows the normalized mean velocity profile versus altitude for three values of the bulk mean velocity. The profiles are rather similar to each other, which implies a linear relationship between flow velocity and friction at the surface. In the surface valleys, we find strongly retarded flow speeds. In the normalized 

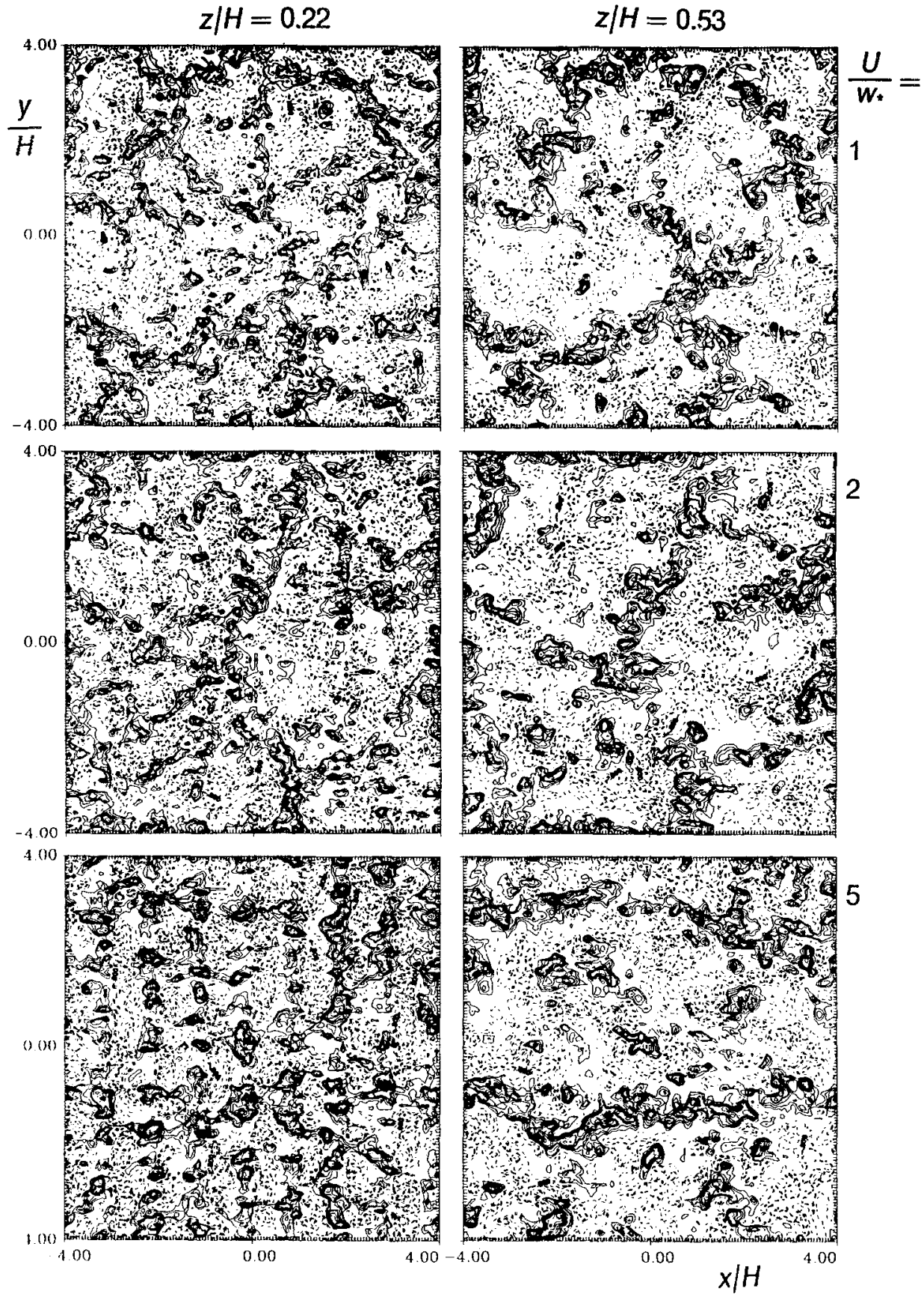

Fig, 11. Flow structure in a horizontal plane at various values of the vertical coordinate (left: $z / H=$ 0.22 , right $z / H=0.53$ ) and for various mean velocities, $U / w_{*}=1$ (top), 2 (middle), 5 (bottom), at $t / t_{*}=24$. Contours represent constant vertical velocity $w$ as in Figure 3. 
(a) $t_{1}$

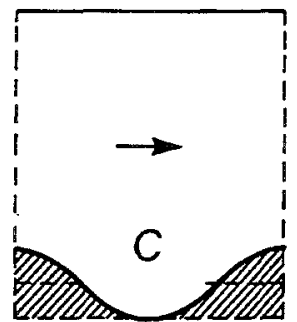

(b) $t_{2}>t_{1}$

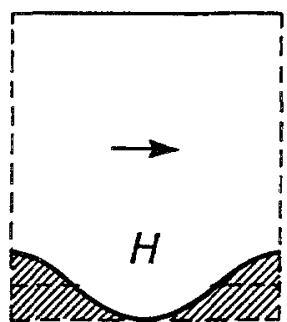

(c) $b_{3}>b_{2}$

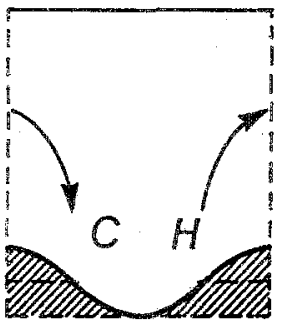

Fig. 12. Sketch showing schematically the formation of a pool of cold fluid (a) which gets heated (b) and leaves the valley as a thermal being replaced by cool air (c), in a quasiperiodic time sequence. $\mathrm{C}=$ cold fluid, $\mathrm{H}=$ hot fluid.
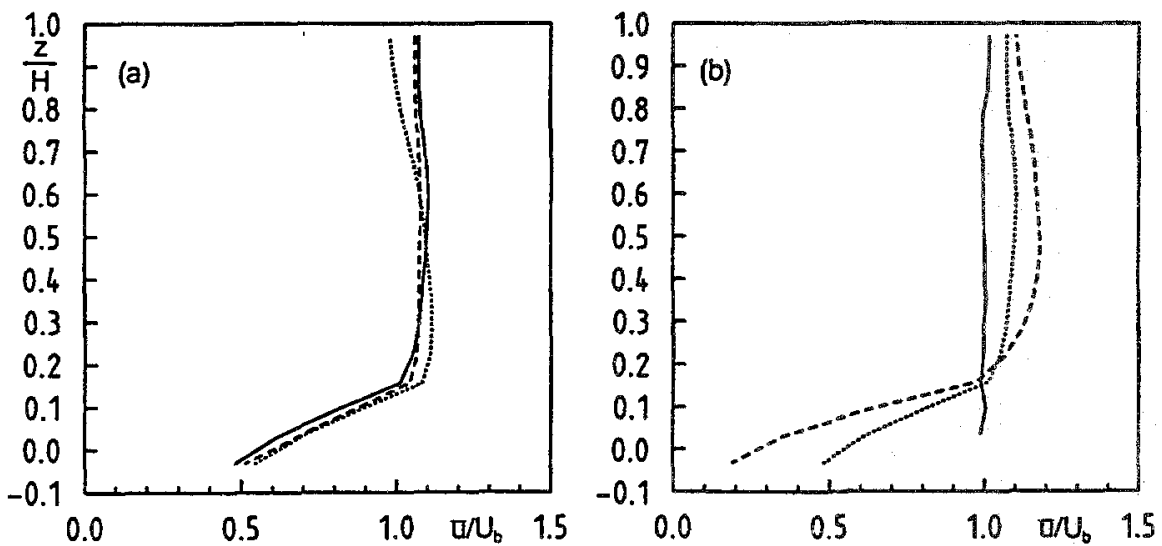

Fig. 13. Mean velocity profile $\bar{u} / U_{b}$ versus vertical coordinate $z / H$ in the time interval $28.5 \leqslant t / t_{*}$ $\leqslant 33.5$, (a) for various mean velocities $U / w_{*}=$ 1 , 0 , 2 , 5. (b) for different amplitude of the surface wave, $\delta / H=$ 0.1 0.15 .

profile, the effect of surface drag is strongest for the smallest mean velocity. Above the crests, the layer is well mixed with uniform velocity except for $U / w_{*} \leqslant 2$, for which we find a pronounced velocity maximum. This overshooting is different from the local maximum in downstream velocity which forms very close to the surface just above the wave crests. The maximum in the mean velocity profile is caused by strong updrafts carrying fluid with low downstream velocity quickly up to the top boundary from where the fluid exchanges momentum with the faster fluid at mid-levels by sinking motion or small-scale turbulence. Hence, this countergradient momentum flux has the same origin as the countergradient heat flux which was indicated by the increasing temperature profile; see Figure 6 . As shown in Schumann (1993), the formation of countergradient fluxes requires either a nonsteady mean profile, as is the case for temperature, or a uniform source. For velocity, the uniform source is due to the mean pressure gradient driving the 

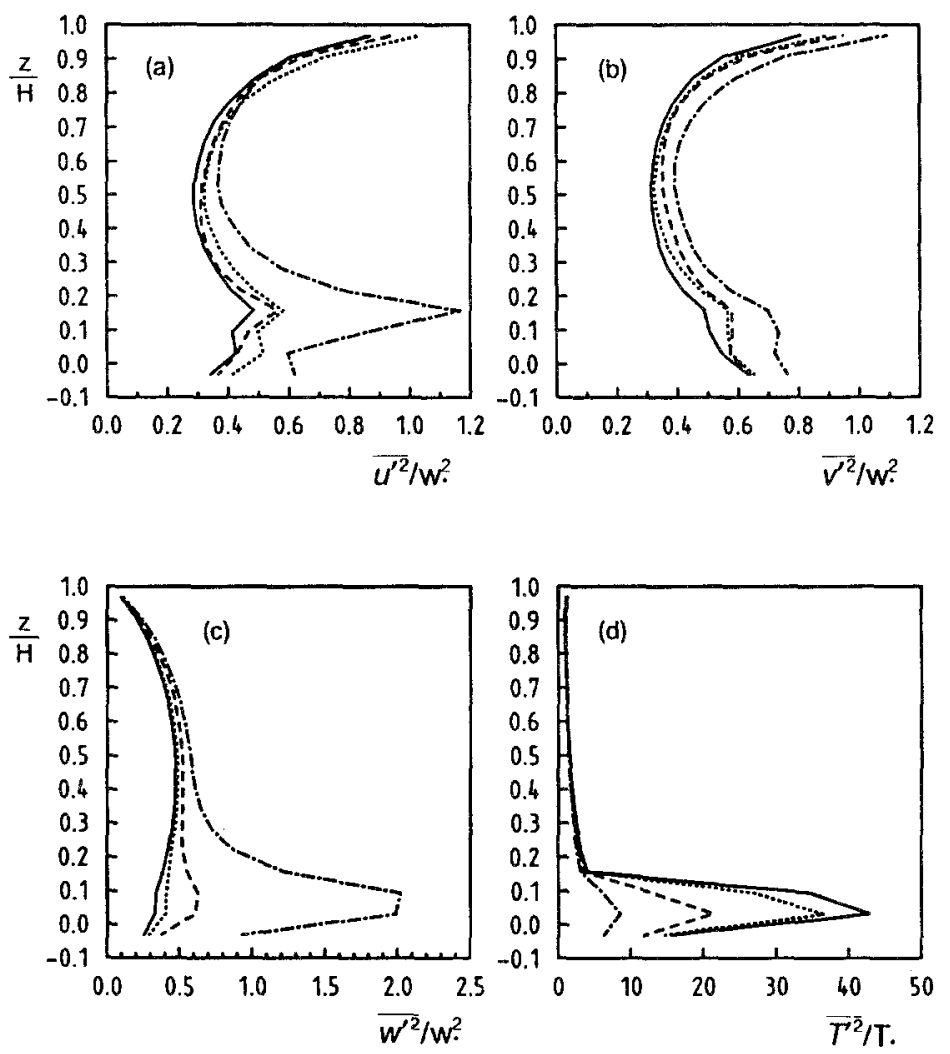

Fig. 14. Effect of mean velocity $U$ on vertical profiles of turbulence variances versus $z / H$ averaged in the time interval $28.5 \leqslant t / t_{*} \leqslant 33.5$ for $\lambda / H=1$. The curves are for $U / w_{*}=$ $0, \ldots 1$, $--2, \ldots-5$. (a) Downstream velocity $\overline{u^{\prime 2}}$, (b) cross-stream velocity $\frac{*}{v^{\prime 2}}, \overline{\text { (c) vertical velocity }}$ $\overline{w^{\prime 2}}$, (d) resolved temperature fluctuations $\overline{T^{\prime 2}}$.

flow in the downstream direction. Obviously, the velocity profiles differ from a logarithmic shape.

As can be seen from Figures $9 \mathrm{a}$ and $\mathrm{b}$, the phase-averaged velocities look not much different for increased mean velocity relative to the reference case, Figure $9 \mathrm{c}$. In particular, the vertical scale of the impact of undulating surfaces remains unchanged, at least for $\lambda=H$. Perhaps the local maximum in $u$ just above the crests becomes more pronounced when $U$ increases.

Figure 14 shows the impact of flow speed on the turbulence variances. The shear enforces a strong increase of downstream and vertical velocity fluctuations for $U / w_{*}>2$. The changes are largest at the level of the wave crests as to be expected due to the kinematical effects of the surface. The changes decrease above and they are small in the upper half of the mixed layer. However, the lateral variance, $\overline{v^{\prime 2}}$, increases strongly also in the upper part of the boundary layer. This is obviously a consequence of downstream rolls which extend vertically over the 


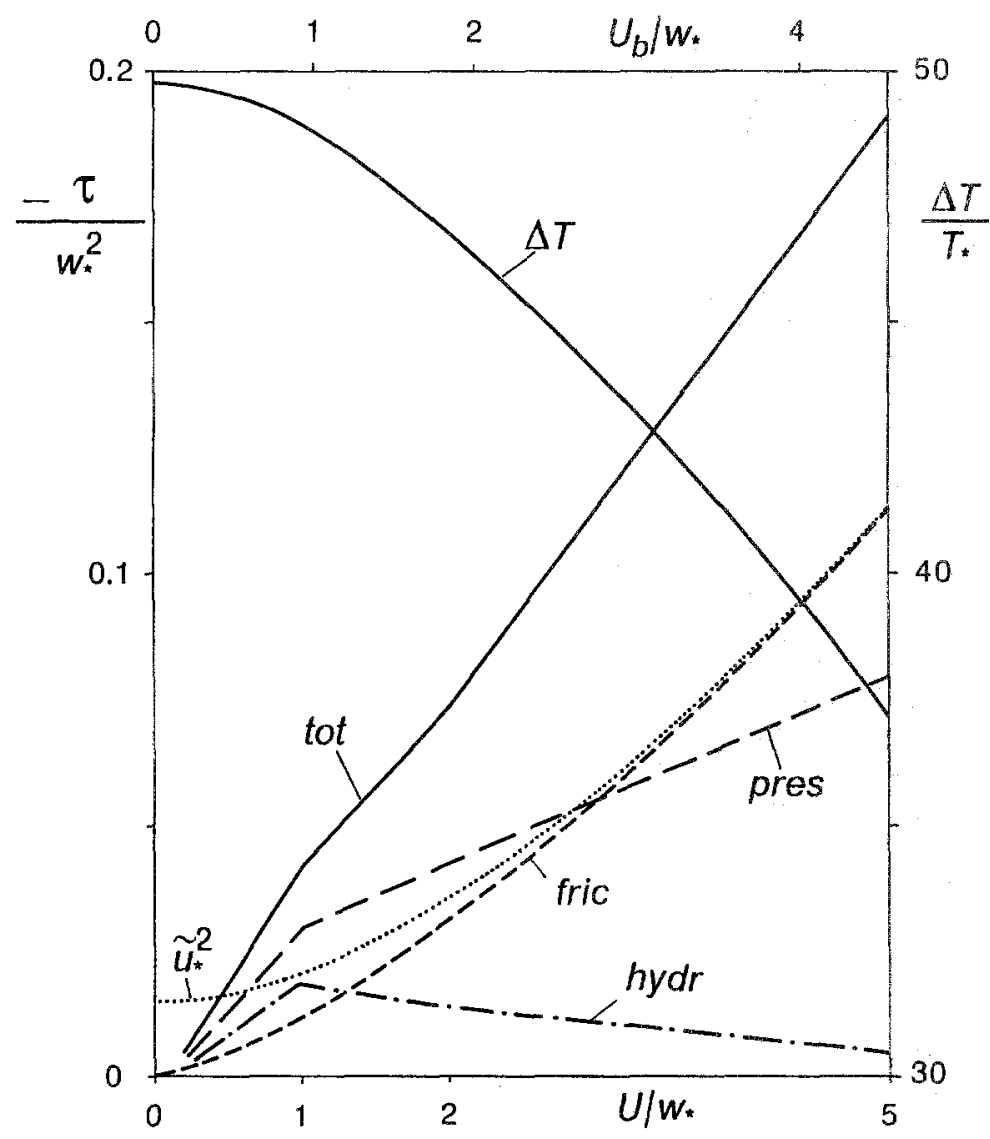

Fig. 15. Momentum flux (left ordinate) and temperature difference (right ordinate) at the surface versus mean velocity $U / w_{*}$ (lower scale) and bulk mean velocity $U_{b} / w_{*}$ (upper scale), for $\lambda / H=1$, $\delta / H=0.1, z_{0} / H=10^{-4}$. Full curves: total flux and temperature difference between surface and bulk, long dashed curve: pressure drag, dash-dotted: hydrostatic part of pressure drag, short dashed: frictional drag, dotted: square of the effective local friction velocity $\tilde{u}_{*}$.

whole layer causing increased cross-stream motions near the upper and lower boundary. The temperature fluctuations (Figure 14d shows the resolved fluctuations without SGS contributions) are virtually unchanged above the terrain peaks. The large temperature fluctuations within the valleys decrease with flow speed because of reduced importance of slope convection.

As shown in Figure 15, both the frictional drag and the pressure forces increase with the mean velocity. The hydrostatic part increases with $U$ between 0 and $w_{*}$ but then decreases slowly with the mean velocity. To first approximation, the total drag increases about linearly with flow speed, as expected from the similarity of velocity profiles; see Figure 13a. The friction part increases with power 1.4 of $U / w_{*}$ and the pressure part with power 0.6. They increase slower than quadratically because the turbulence is mainly of buoyant origin and hence only slightly 
dependent on shear. A quadratic increase is to be expected for $U \gg w_{*}$. However, shear becomes important already for $U / w_{*}>2.5$, for which $u_{*} / w_{*}>0.35$; for $U / w_{*}=5$, we find $u_{*} / w_{*}=0.44$. Hence, the absence of rolls for $U / w_{*}=2$, and the presence of longitudinal rolls for $U / w_{*}=5$ are again consistent with the findings of Sykes and Henn (1989).

Figure 15 also shows the square value of an effective friction velocity $\tilde{u}_{*}$, which is computed from the local frictional momentum flux. The square of this friction velocity is larger than the negative frictional flux because of the contributions from turbulent fluctuations in both horizontal directions near the lower surface. For $U=0, \bar{u}_{*}$ represents the minimum friction velocity as computed by Schumann (1988) and Schmidt and Schumann (1989). For $U / w_{*}>2$, it approaches the value of the friction velocity which results from the diffusive part of the flux of downstream momentum at the surface. The nonzero value of $\tilde{u}_{*}$ causes finite heat transfer rate even for zero mean velocity. The temperature difference between the surface and the bulk of the fluid is also listed in Table I for all cases and is plotted versus mean velocity in Figure 15. It is fairly insensitive to mean motions for $U<w_{*}$, and decreases for larger mean velocities. The temperature difference is about 3\% greater than that calculated from the Monin-Obukhov relationships for flat surfaces and steady mean velocity $\left(U_{b}^{2}+\overline{u^{\prime 2}}\right)^{1 / 2}$ at $z=0.1 H$. Hence, the surface undulation has little impact on the transfer of scalar quantities.

(b) Amplitude of the surface wave: $\delta / H=0,0.1,0.15$.

The flow structure changes if the amplitude is increased above the value 0.1 considered before (see Figure 16) and shows a weak tendency to form downstream rolls. This is most obvious at midlevels in the CBL whereas the flow structure in the surface layer is still rather irregular with many small-scale convection lines. Corresponding plots at $z / H=0.78$ are very similar to those at midlevels with little indication of downstream tilt of the vertical structures. The rolls for $\delta / H=$ 0.15 are again a consequence of strong shear. In fact, from Table $I$, for this amplitude, we compute $u_{*} / w_{*}=0.31$, a value close to the expected limit for the creation of rolls. Figure $13 \mathrm{~b}$ shows that the rougher surface causes stronger shear in the lower part of the boundary layer. We again observe an overshooting in the mean velocity profile with negative mean velocity gradient in the upper part. This effect increases with surface slope, suggesting that strong updrafts are enhanced by upward motions at the slopes. For the flat surface, Figure $13 \mathrm{~b}$ shows a uniform velocity profile down to the lowest grid point. This grid point lies above the Obukhov scale, $0.005 H$ in this case, so that shear is important only very close to the surface.

From Figure $9 \mathrm{~g}$, for $\delta / H=0.15$, it appears as if the mean flow field is close to forming a recirculation zone in that $u$ approaches zero at the floor of the valley. Also Figure $10 \mathrm{~g}$ shows that $w$ is close to zero over a relatively wide part of the bottom of the valley. However, a steady recirculation zone is not formed even for 
this steep slope. Recirculations occur only locally in the instantaneous velocity fields. The non-separated mean flow was not expected for a slope angle of $43^{\circ}$. Studies for neutral boundary layers (Buckles et al., 1984; Mason and King, 1984) found recirculations for smaller slope angles.

According to Taylor et al. (1989) and Hunt et al. (1991), for neutral static stability, the form drag is proportional to the square of the maximum slope steepness $2 \pi \delta / \lambda$, as long as any separated flow is confined to a narrow region, but the drag grows only linearly with $2 \pi \delta / \lambda$ when $\delta / H>0.1$ and the flow separates. If one plots the values of momentum flux, as given in Table $\mathbb{I}$, as a function of $2 \pi \delta / \lambda$, one finds a strong increase of pressure drag with slope steepness. The increase is, however, different from quadratic because of changing contributions from hydrostatic and dynamic pressure forces and strong buoyant mixing. Only the two data points for $\delta=0.15 H$ and the corresponding one for $\delta=0.1 H$ support a quadratic increase. The total drag exceeds the value for flat surfaces by a factor of two for a slope steepness of about 0.4 and increases strongly for larger values of slope steepness. The limit 0.4 certainly depends upon the ratio $z_{0} / \lambda$. For pure shear layers, the normalized form drag coefficient $A_{p}=\tau_{\text {dyn }} /\left[\tau_{0}(2 \pi \delta / \lambda)^{2}\right]$ should be a weak function of $\lambda / z_{0}$. Here, $\tau_{0}$, is the friction drag for the same wind speed over the flat surface. Our results show a variation of this coefficient between 0.3 and 4 for a relatively small variation of $\lambda / z_{0}$ between $10^{4}$ and $4 \cdot 10^{4}$. In contrast, Figure 2 of Taylor et al. (1989) suggests that this value should be less variable and close to a value of order 10 . The discrepancy is caused by the buoyant turbulence.

The lateral temperature differences between updrafts and downdrafts are of order $T_{*}$ (Schumann, 1991b). The plumes extend over the whole depth of the $\mathrm{CBL}$ and therefore induce horizontal variations in hydrostatic surface pressure of order $\rho \beta g H T_{*}=\rho w_{*}^{2}$. A net force from the pressure fluctuations on the surface requires a phase shift between the hydrostatic pressure variations at the surface and the surface height. The shift will grow with $U / w_{*}$ and $H / \lambda$, but the intensity of the coherent motions and the resultant pressure variations will presumably decrease with the same parameters. In any case, the downstream force from the hydrostatic pressure variations depends upon the slope and therefore causes a drag of order $w_{*}^{2} \delta / \lambda$. This should be valid at least for wavelengths of the order of the horizontal scale of the convective motion in the CBL, i.e., for scales of order $4 H$. In contrast, the dynamic (form) pressure drag grows with $U^{2}(\delta / \lambda)^{2}$, so that buoyant hydrostatic forces are important as long as $\left(U / w_{*}\right)^{2} \delta / \lambda$ is small. From Table I, we find that the hydrostatic part is larger than the dynamic part if this number is less than about 0.15. Taylor (1977) and Mason (1987) assume that the flow above the wave crests and above the thermal surface layer is horizontally isothermal so that the hydrostatic forces scale with this smaller height rather than with the depth of the CBL.

The frictional drag becomes smaller when $\delta / H$ increases. As a consequence, the surface temperature difference $\Delta T$, see Table I, over steep terrain is about $4 \%$ larger than for flat terrain. For fixed mean velocity $U=w_{*}$, and $\delta / H=0.1$, but 

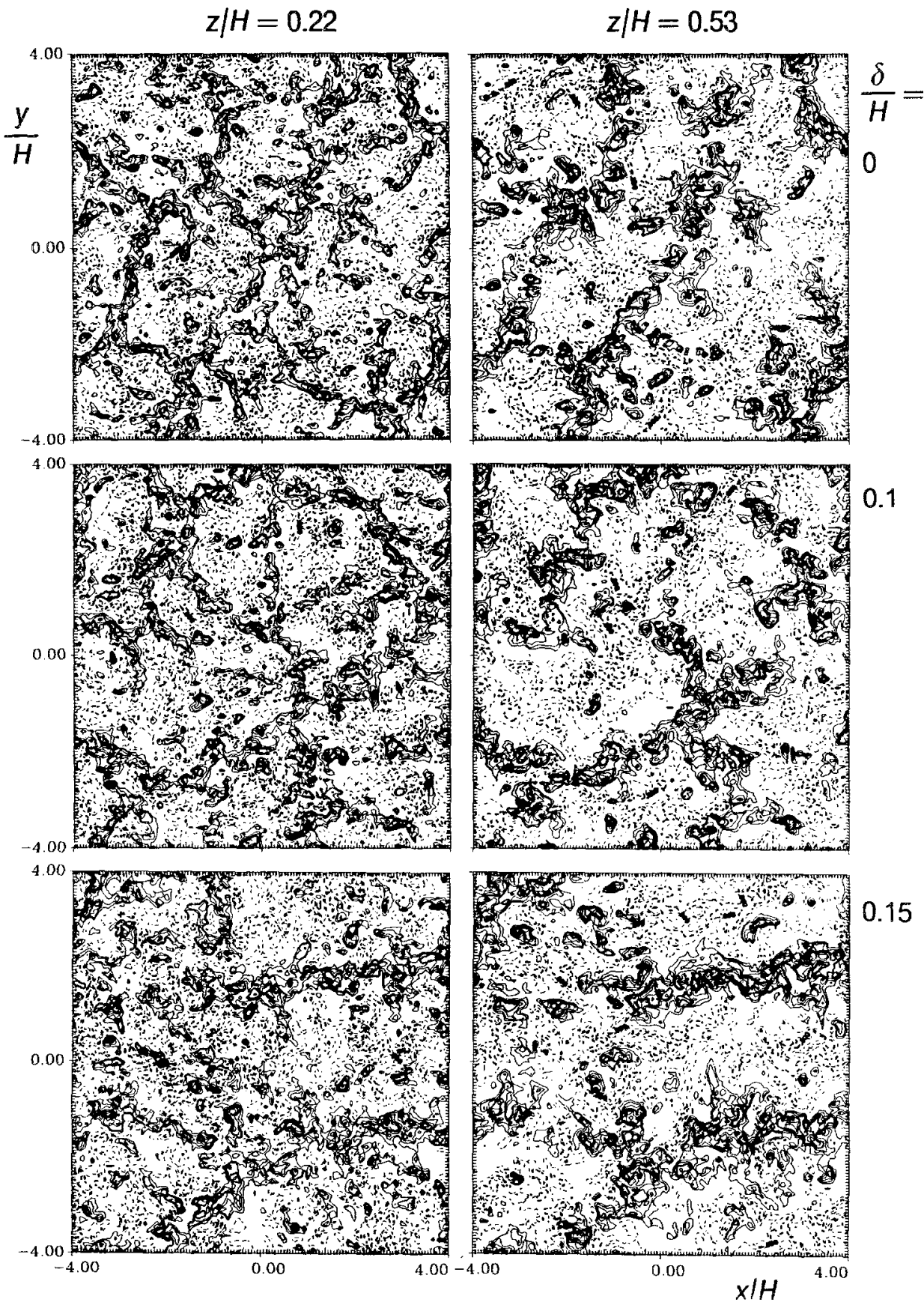

Fig. 16. The flow structure in a horizontal plane at various values of the vertical coordinate (left: $z / H=0.22$, right: $z / H=0.53$ ) and for various surface amplitudes $\delta / H=0$ (top), 0.1 (middle), 0.15 (bottom), at $t / t_{*}=24$. Contours represent constant vertical velocity $w$ as in Figure 3 . 
for varying wavelengths, $\Delta T$ is at most $2 \%$ larger than for a flat surface. Hence, the wavy terrain reduces the heat transfer only slightly.

\section{Discussion and Conclusions}

This study has determined the impact of a wavy surface on the CBL at weak to moderate mean winds perpendicular to the surface wave crests. For weak mean winds of order $w_{*}$, the convective structure is similar to that over flat terrain (Schmidt and Schumann, 1989) but the polygonal structures near the surface form only as half-rings and are disrupted on the downstream side. As for zero mean wind, isolated plumes develop at mid-levels of the CBL. Weak mean wind tends to destroy any coherent roll motions induced by the undulating surface for zero mean wind (Krettenauer and Schumann, 1992) and therefore reduces the impact of terrain parameters on the turbulence statistics. For stronger mean wind, the turbulence variances increase. For short wavelengths, this increase is limited to levels below $\lambda /(2 \pi)$. Longitudinal rolls are found for $u_{*} / w_{*}$ larger than about 0.35 , in agreement with Sykes and Henn (1989). In the set of cases which we have considered, such large friction velocities arise either for strong enough mean wind $\left(U / w_{*}>2.5\right)$ or steep surface waves $(\delta / \lambda \geqslant 0.15)$. The longitudinal rolls cause enhanced lateral turbulence fluctuations also in the upper part of the CBL. The convective structures have dominant scales of the order $4 H$ in the horizontal directions. However, it requires rather long times of order $20 \mathrm{H} / \mathrm{w}_{*}$ to develop such structures; thus they might be of little relevance for atmospheric cases with nonstationary boundary conditions. For shorter times, the direct influence of smallscale surface properties is greater.

None of the cases shows a recirculating flow region in the mean fields. Instead we found a quasi-periodic formation of pools of stagnant cold fluid in the valleys which become heated and then rise as bubbles. A stationary recirculation zone appears not to exist for the bottom-heated boundary layer. Such a zone can exist only if the heat flux from the surface into the recirculating fluid is balanced by turbulent transfer of heat out of this zone across the bounding streamline with zero motion. However, the turbulent flux of heat from the recirculation zone to the mixed layer above must be small, because otherwise the turbulence would also carry a strong momentum flux which accelerates and erodes the separated region. Hence, the recirculating fluid becomes warmer and more buoyant until it finally leaves the valley as a thermal, destroying the recirculation.

The surface drag is composed of friction and pressure forces. The frictional drag over the wavy surface is a little smaller than over flat surfaces. As a consequence, the heat transfer rate is diminished, causing increased temperature differences for constant flux. The pressure drag originates from hydrostatic and dynamic forces. The hydrostatic part is caused by temperature differences within the layer as related to updrafts and downdrafts, and it is rather large for short wavelengths and small wind speeds. The dynamic part is the classical form drag which grows 
approximately quadratically with flow speed and wave steepness, at least for strong mean wind. It exceeds the hydrostatic part for about $\left(U / w_{*}\right)^{2} \delta / \lambda>0.15$. The mean profiles of velocity and temperature deviate strongly from a logarithmic shape so that an unique effective roughness length does not exist for strongly convective conditions. The profiles exhibit, moreover, strong countergradient fluxes in the upper half which shows the failure of the gradient-driven mixing concept in the whole CBL.

The LES sufficiently resolves all relevant scales within the CBL above the surface layer. In particular, a domain with a lateral side-length of $8 H$ and a mesh with spacings of $H / 16$ appears to be sufficient for this purpose. The effect of short surface waves could hardly be represented by a statistical turbulence model which requires the turbulence scales to be smaller than the geometrical scales. However, as discussed in the Appendix, the present study does not resolve the very thin surface layer in which the conditions for surface-layer similarity are strictly satisfied. As a consequence, surface friction is represented only approximately. The resultant approximation errors may be important and of the order $20 \%$, in particular for the computed surface drag. However, the distortion imposed by the undulating surface on the mean flow and the turbulence in the inner part of the mixed layer will be less affected by this limitation. Also, it is questionable whether previous estimates of the required vertical resolution apply for the convective cases, where the flow behaves much differently from the classical boundary-layer concept and where the surface layer is strongly dependent on vertical and lateral mixing within the CBL. Although the scope of variations was small, the parameter studies showing only weak influences from the limited grid resolution corroborate the conjecture that the details of the surface layer are of little importance for this study. Unfortunately, no information exists from field or laboratory investigations which would be suitable to check the accuracy of the simulation results. Such experiments should be performed.

With respect to atmospheric boundary layers over hilly terrain, the study is of an exploratory nature only, because we have not treated realistic land surface shapes and have assumed uniform surface heating. However, the immense variety of real surface forms and heat flux distributions cannot be covered with a few simulations. Moreover, the top boundary does not account for entrainment at a real inversion, which contributes to friction of internal motion modes. This suggests that the variances are overestimated in the upper part of the CBL. On the other hand, the CBL might excite wavy motions in the troposphere above the inversion and that could feed back to enhanced large-scale motions in the CBL causing larger variances. Further investigations are required to identify the magnitude of such effects. The LES method is a suitable basis for such purposes. 


\section{Appendix: Vertical Resolution Requirement for the Surface Layer}

Based on the theory for neutral boundary-layer flows over low hills without recirculations, Hunt et al. (1988) divide the flow above a hill into two layers: an inner layer where the turbulence is in local equilibrium with the local shear stress, and an outer layer where the response of the flow to the hill is essentially controlled by the large-scale dynamics. For neutral stability, the thickness of the inner layer $l$ satisfies approximately $(l / \lambda) \ln \left(l / z_{0}\right)=\kappa^{2} / 2$, where $\kappa$ is the von Karman constant (we have identified $\lambda / 4$ with the half-width used by Hunt et al.). For our reference case, this estimate gives a value of $l / H$ of about 0.01 . It would imply that about 50 grid points are necessary as a minimum in the vertical because our first grid point is $\Delta z / 2$ above the surface. Simulations with such a fine grid would be very expensive. In view of the stronger vertical mixing by convective turbulence, it appears reasonable to assume that the inner layer is considerably thicker for the CBL. The thickness of the surface layer will be estimated below from a simple analysis of vertical mixing and mean motion but ignoring accelerations induced by the terrain.

The thickness of the surface layer $l$ may be defined as that layer over which vertical mixing occurs by the friction velocity $u_{*}$ while the fluid has moved with its flow velocity $U(l)$ over the horizontal scale, say $\lambda / 4$, of the surface mountain,

$$
\frac{l}{\lambda / 4}=\frac{u_{*}}{U(l)} .
$$

For the neutral surface layer, one may estimate $U(l)=\kappa^{-1} u_{*} \ln \left(l / z_{0}\right)$. As a result we obtain

$$
\frac{l}{\lambda}=\frac{\kappa}{4 \ln \left(l / z_{0}\right)} .
$$

This simple estimate gives a value of $l$ which is only 1.25 times larger than that deduced by Hunt et al. (1988).

For the heated surface layer, the logarithmic law may be replaced by the MoninObukhov relationships. We use the same functions as employed in the LES boundary conditions (Schmidt and Schumann, 1989). Moreover, in order to obtain a meaningful result for zero mean velocity, we follow the reasoning of Schumann (1988) and compute the thickness $l$ for the effective velocity $U_{\text {eff }}=\left(U^{2}+\overline{u^{\prime 2}}\right)^{1 / 2}$. The horizontal velocity variance at zero mean wind is estimated (Schumann, 1988) as $\overline{u^{\prime 2}}=0.7^{2} w_{*}^{2}$. From these definitions, the ratio $l / H$ has been evaluated and plotted for various wavelengths in Figure 17. We find that the surface-layer thickness $l$ is larger for pure convection (zero mean velocity) than for pure shear flows (infinite ratio $U / w_{*}$ ) by a factor increasing from 1.7 for $\lambda / H=1$ to 2.0 for $\lambda / H=4$. As expected, the surface-layer thickness is smallest for short wavelengths and large mean velocities and increases about linearly with wavelength $\lambda / H$. Larger values of $z_{0} / H$ would cause larger values of $l$. 


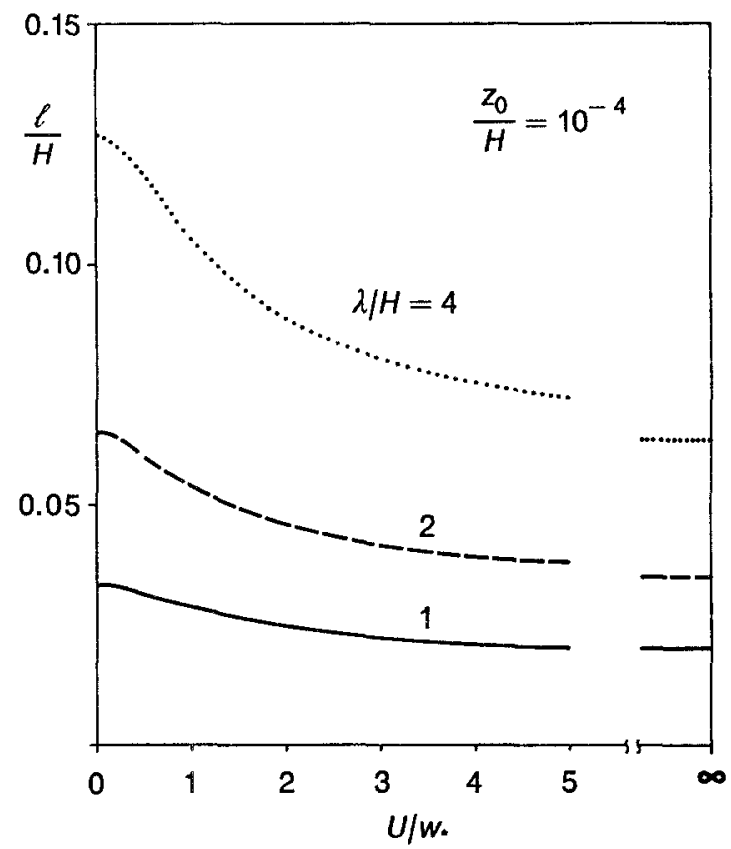

Fig. 17. Estimate of surface-layer thickness $l$ versus wind speed $U(l)$ at the top of the surface layer for gentle surface waves with various wavelengths $\lambda$ and for fixed roughness height $z_{0} / H=10^{-4}$.

If one requires that $\Delta z / 2<l$ and employs an equidistant vertical grid, then the number of grid points in the vertical should be at least 15 for pure convection but 25 for the case with shortest wavelength and largest mean wind considered in this study. Of course, these numbers cannot be more than the estimate of the required magnitude of grid spacings.

The actual requirements may be more or less stringent. More stringent conditions have been posed by Mason and King (1984) who require that the vertical integral of the horizontal pressure gradient and advection terms from the surface to $l_{*}$ is negligible in magnitude compared with the surface stress. Here, $l_{*}$ is the very small scale of surface similarity. They show that $l_{*} \ll C_{d} \lambda$ where $C_{d}$ is a drag coefficient, which is typically of order 0.002. Mason (1987) estimates the same scale from $l_{*} \ln ^{2}\left(l_{*} / z_{0}\right)=\kappa \lambda / \pi$, which gives a similarly small magnitude of $l_{*}$. According to Hunt et al. (1988), the limit of surface-layer similarity is reached when $l_{*} \cong\left(z_{0} l\right)^{1 / 2}$, which is of order $10^{-3} \mathrm{H}$ in our simulations. Also the results of Zeman and Jensen (1987) suggest that the turbulence profile differs strongly from the profiles over flat surfaces below $l$. Obviously, equidistant resolution of the boundary layer at vertical scales of order $l_{*}$ is not possible with present computers. Variable grid levels might be helpful in this respect but do not resolve the smallscale horizontal turbulence variations and increase the computational effort by a need for smaller time steps. 
Less stringent requirements result from replacing $u_{* *}$ by the turbulent fluctuations at height $l$. The vertical velocity fluctuations near the surface increase as $w_{*}(l / H)^{1 / 3}$, which is larger than $u_{*}$ for small values of $l / H$. Moreover, it should be noted that the surface-layer dynamics in the purely convective layer, for given surface heating, is virtually independent of the surface friction but strongly controlled by exchange processes between the surface layer and the mixed layer (Schumann, 1988). Also, an LES of the CBL with only 10 grid layers within the boundary layer has been shown to give results very similar to those for 40 equidistant grid layers (Schumann, 1991a) and results obtained with variable vertical grid spacings (Nieuwstadt et al., 1992). Finally, the results of Brutsaert and Sugita (1990) affirm the applicability of the Monin-Obukhov relationship to the CBL up to about $z / H=0.1$ over wavy terrain for a wavelength comparable to the depth of the CBL. Therefore, it might be possible that previous estimates of the required resolution near the surface are too restrictive.

\section{References}

Brutsaert, W. and Kustas, W. P.: 1987, 'Surface Water Vapor and Momentum Fluxes under Unstable Conditions from a Rugged-Complex Area', J. Atmos. Sci. 44, 421-431.

Brutsaert, W. and Sugita, M.: 1990, 'The Extent of the Unstable Monin-Obukhov Layer for Temperature and Humidity above Complex Hilly Grassland', Boundary-Layer Meteorol. 51, 383-400.

Buckles, J., Hanratty, T. J., and Adrian, R. J.: 1984, 'Turbulent Flow over Large-Amplitude Wavy Surfaces', J. Fluid Mech. 140, 27-44.

Druilhet, A., Noilhan, J., Benech, B., Dubosclard, G., Guedalia, D., and Frangi, J.: 1983, 'Étude expérimentale de la couche limite au-dessus d'un relief modérè proche d'une chaîne de montagne', Boundary-Layer Meteorol. 25, 3-16.

Emeis, S.: 1990, 'Pressure Drag of Obstacles in the Atmospheric Boundary Layer', J. Appl. Meteorol. 29. $461-476$.

Grant, A. L. M. and Mason, P. J.: 1990, 'Observations of Boundary-Layer Structure over Complex Terrain', Quart. J. R. Meteorol. Soc. 116, 159-186.

Hadfield, M. G., Cotton, W. R., and Pielke, R. A.: 1991, 'Large-Eddy Simulations of Thermally Forced Circulations in the Convective Boundary Layer. Part I. A Small-Scale Circulation with Zero Wind', Boundary-Layer Meteorol. 57, 79-114.

Hadfield, M. G., Cotton, W. R., and Pielke, R. A.: 1992, 'Large-Eddy Simulations of Thermally Forced Circulations in the Convective Boundary Layer. Part II. The Effect of Changes in Wavelength and Wind Speed', Boundary-Layer Meteorol. 58, 307-327.

Hechtel, L. M., Moeng, C.-H., and Stull, R. B.: 1990, 'The Effects of Nonhomogeneous Surface Fluxes on the Convective Boundary Layer: A Case Study Using Large-Eddy Simutation', J. Atmos. Sci. 47, 1721-1741.

Hunt, J. C. R., Leibovich, S., and Richards, K. J.: 1988, 'Turbulent Shear Flows over Low Hills', Quart. J. R. Meteorol. Soc. 114, 1435-1470.

Hunt, J. C. R., Tampieri, F., Weng, W. S., and Carruthers, D. J.: 1991, 'Air Flow and Turbulence over Complex Terrain: a Colloquium and a Computational Workshop', J. Fluid Mech. 227, 667688.

Huynh, B. P., Coulman, C. E., and Turner, T. R.: 1990, Some Turbulence Characteristics of Convectively Mixed Layers over Rugged and Homogeneous Terrain', Boundary-Layer Meteorol. 51, $229-254$.

Kaimal, J. C., Eversole, R. A., Lenschow, D. H., Stankow, B. B., Kahn P. H., and Businger, J. A.: 1982, 'Spectral Characteristics of the Convective Boundary Layer over Unevell Terrain', I. Atmos. Sci. 39, 1098-1114. 
Krettenauer, K., and Schumann, U.: 1989, 'Direct Numerical Simulation of Thermal Convection over a Wavy Surface', Meteorol. Atmos. Phys. 41, 165-179.

Krettenauer, K., and Schumann, U.: 1992, 'Numerical Simulation of Turbulent Convection over Wavy Terrain', J. Fluid Mech. 237, 261-299.

Krishnamurti, R., and Howard, L. N.: 1981, 'Large-scale Flow Generation in Turbulent Convection', Proc. Natl. Acad. Sci. USA 78, 1981-1985.

Mason, P. J.: 1987, 'Diurnal Variations in Flow over a Succession of Ridges and Valleys', Quart. $J$. R. Meteorol. Soc. 113, 1117-1140.

Mason, P. J., and King, J. C.: 1984, 'Atmospheric Flow over a Succession of Nearly Two-Dimensional Ridges and Valleys', Quart. J. R. Meteorol. Soc. 110, 821-845.

Nieuwstadt, F. T. M., Mason, P. J., Moeng, C.-H., and Schumann, U.: 1992, 'Large-Eddy Simulation of the Convective Boundary Layer: A Comparison of Four Computer Codes', in F. Furst et al. (eds.), Turbulent Shear Flows 8, Springer, 343-368.

Raasch, S., and Groß, G.: 1992, 'VISES: Visualisierung numerischer Simulationsergebnisse mittels Videofilm', Meteorol. Z., 1, 211.

Raupach, M. R., Weng, W. S., Carruthers, and Hunt, J. C. R.: 1992, 'Temperature and Humidity Fields and Fluxes over Low Hills', Quart. J. R. Meteorol. Soc. 118, 191-225.

Schmidt, H., and Schumann, U.: 1989, 'Coherent Structure of the Convective Boundary Layer Deduced from Large-Eddy Simulation', J. Fluid Mech. 200, 511-562.

Schumann, U.: 1988, 'Minimum Friction Velocity and Heat Transfer in the Rough Surface Layer of a Convective Boundary Layer', Boundary-Layer Meteorol. 44, 311-326.

Schumann, U.: 1990, 'Large-Eddy Simulation of the Up-Slope Boundary Layer', Quart. J. R. Meteorol. Soc. 116, 637-670.

Schumann, U.: 1991a, 'Subgrid Length-Scales for Large-Eddy Simulation of Stratified Turbulence', Theor. Comput. Fluid Dyn. 2, 279-290.

Schumann, U.: 1991b, 'A Simple Model of the Convective Boundary Layer over Wavy Terrain with Variable Heat Flux', Beitr. Phys. Atmosph. 64, 169-184.

Schumann, U.: 1993, 'Transport Asymmetry in Skewed Convective Circulations', J. Atmos. Sci. 50, 116-119.

Scorer, R. S.: 1978, Environmental Aerodynamics, J. Wiley.

Sykes, R. I. and Henn, D. S.: 1989, 'Large-Eddy Simulation of Turbulent Sheared Convection', J. Atmos. Sci. 46, 1106-1118.

Tampieri, F.: 1987, 'Separation Features of Boundary-Layer Flow over Valleys', Boundary-Layer Meteorol. 40, 295-307.

Taylor, P. A.: 1977, 'Some Numerical Studies of Surface Boundary-Layer Flow above Gentle Topography', Boundary-Layer Meteorol. 11, 439-465.

Taylor, P. A., Mason, P. J., and Bradley, E. F.: 1987, 'Boundary-Layer Flow over Low Hills', Boundary-Layer Meteorol. 39, 107-132.

Taylor, P. A., Sykes, R. I., and Mason, P. J.: 1989, 'On the Parameterization of Drag over SmallScale 'Topography in Neutrally-Stratified Boundary-Layer Flow', Boundary-Layer Meteorol. 48, 409422.

Walko, R. L., Cotton, W. R., and Pielke, R. A.: 1992, 'Large-Eddy Simulations of the Effects of Hilly Terrain on the Convective Boundary Layer', Boundary-Layer Meteorol. 58, 133-150.

Zeman, O. and Jensen, N. O.:1987, 'Modification of Turbulence Characteristics in Flow over Hills', Quart. J. R. Meteorol. Soc. 113, 55-80. 